\title{
Flow Characteristics and Fluid Forces Reduction of Flow Past Two Tandem Cylinders in Presence of Attached Splitter Plate
}

\author{
Ali Ahmed (iD, ${ }^{1}$ Abdul Wahid, ${ }^{2}$ Raheela Manzoor, ${ }^{3}$ Noreen Nadeem, ${ }^{3}$ Naqib Ullah, ${ }^{4}$ \\ and Shazia Kalsoom ${ }^{3}$ \\ ${ }^{1}$ Department of Mathematics, COMSATS University Islamabad, Park Road, Tarlai Kalan 45550, Islamabad, Pakistan \\ ${ }^{2}$ Balochistan University of Information Technology Engineering and Management Science, Quetta, Balochistan, Pakistan \\ ${ }^{3}$ Department of Mathematics, Sardar Bahadur Khan Women University, Quetta, Balochistan, Pakistan \\ ${ }^{4}$ University of Central Punjab Islamabad Campus, Islamabad, Pakistan
}

Correspondence should be addressed to Ali Ahmed; alihmd87@gmail.com

Received 15 April 2021; Accepted 19 July 2021; Published 8 September 2021

Academic Editor: Irfan Kaymaz

Copyright (c) 2021 Ali Ahmed et al. This is an open access article distributed under the Creative Commons Attribution License, which permits unrestricted use, distribution, and reproduction in any medium, provided the original work is properly cited.

\begin{abstract}
Numerical simulations are carried out to study the flow around two tandem square cylinders (SC) under the effect of spacing ratio $(g / D)$ and splitter plate length $(l / D)$ for a fixed Reynolds number $(\operatorname{Re})=100$. The $g / D$ is varied from 0 to 10 and $l / D$ is varied from 0.5 to 10 . The splitter plate length is found to have strong effect on vortex shedding and fluid forces. The maximum reduction in mean drag coefficient is observed at $l / D=8$, that is $15 \%$ and $78 \%$ for upstream and downstream cylinders, respectively. The maximum reduction in root-mean-square value of lift coefficient is found at $l / D=10$, that is $99 \%$. The flow pattern at both of these points is steady flow. There is $100 \%$ vortex shedding suppression for $l / D>5$. The observed flow patterns for flow past tandem cylinders without splitter plate are; single bluff body (SBB), steady flow (SF), quasi-steady flow (QSF), fully developed flow (FDF) and fully developed two-row vortex street flow (FDTRVS) regimes. SBB, QSF and SF regimes were observed in presence of splitter plate.
\end{abstract}

\section{Introduction}

The study of fluid forces and vorticity dynamics through the bluff body has been greatly improved in the last few decades. Most of these studies have been taken for single cylinder. However, much less attention has been paid to multiple cylinders flow compared to single cylinder. The addition of another bluff body can change the different aspects of flow such as fluid forces and transition threshold. To study the flow interference different models such as side-by-side, tandem and staggered models have been used. Among these models, tandem is the simplest one. A number of experimental and numerical studies have been conducted for flow past tandem cylinders. Kondo and Matsukuma [1] numerically analyzed the salient flow features through two tandem circular cylinders at $\mathrm{Re}=1000$. They found that the forces exerted on the last cylinder are substantially higher than those exerted on the first cylinder. While in case of tandem square cylinders, the upstream cylinder forces are larger than those of the downstream cylinder [2]. Azuma et al. [3] experimentally measured forces acting on square structures both side-by-side and in tandem. They observed that the drag forces of two tandem cylinders changed significantly when the separation ratio was greater than $g / D=3$ (where $g$ is the spacing between cylinders and $D$ is cylinder diameter) as compared to side-by-side arrangement. Abbasi et al. [4] numerically analyzed the flow regime transitions over the flow around three tandem cylinders for the $g / D$ between 0.5 and 16 at $R e=200$ and found five different types of flow regimes. In addition, they noted that the $g / D$ is the important analysis parameter. Abbasi et al. [5] analyzed the flow regime transitions around two-, three- and four-tandem cylinders for Re ranging from 1 to 130 at $g / D=2$ and 5 . They observed two separate trends in terms of flow transitions for $g / D=2$ and 5 . The more reduction in terms of drag is observed at $g / D=2$. Mittal et al. [6] numerically 
analyzed the flow characteristics through two cylinders for $\operatorname{Re}=100$ and 1000 and found that the shedding starts at $\operatorname{Re}=100$. Abbasi et al. [7] numerically found three various types of flow features for two tandem cylinders. Vikram et al. [8] also analyzed the salient flow features past tandem cylinders. They observed that compared to downstream cylinder the upstream cylinder experience higher lift force. Sharman et al. [9] numerically analyzed the flow through two inline cylinders under the influence of $g / D$ varying from 2 to 10 for $\operatorname{Re}=100$. They identified $g / D=3.75-4$ as critical spacing, where the flow characteristics changes abruptly. Liu and Jerry [10] experimentally analyzed flow on two cylinders in tandem with $\mathrm{Re}=2000-16000$ and $g / D=1.5-9$. They reported hysteresis with two discontinuous jumps associated with different flow regimes. Sohankar and Etminan [11] analyzed flow and heat transfer across cylinders arranged inline for $R e=1-200$. They found that vortex shedding formation begins in the range $35 \leq \operatorname{Re} \leq 40$.

The addition of an eddy promoter improved the heat transfer of the downstream cylinder and controls the $C_{D}$. Bearman $[12,13]$ used the splitter plates to increase the formation length and reduce the drag. One of the most important studies was carried out by Zdravkovich [14], which divided the vortex suppression into three different branches, namely: (i) surface protrusions, (ii) shrouds and (iii) near-wake stabilizers. In literature both active and passive methods exist. Active control procedures generally require continuous external energy to reduce the drag forces and improve the lift $[15,16]$. While for the passive methods there is no need internal energy, these include tripping rods [17], control cylinder [18-20], splitter plate [21], T-shaped plate [22] etc.

Anderson and Szewczyk [23] experimentally analyzed the impacts of low length splitter plates for the flow through circular cylinder and noticed that less than $D / 8$ splitter length appreciably affect the wake zone for $\mathrm{Re}=2700$ to 46,000 . You et al. [24] observed that significant noise reduction can take place for flow around circular cylinder once the splitter plate length is identical to the cylinder diameter. Uffinger et al. [25] analyzed the effects of different shapes on flow control behind a cylinder and found that the wedge shape behind the square body significantly control the flow. Ali et al. [26] computationally analyzed the influence of splitter plate length on a cylinder at $\mathrm{Re}=150$.

To the best of ours' knowledge, for the proposed problem there is no numerical and experimental study reported in the literature. Hopefully, this work will help engineer's working on fluid forces reduction. The main motivation is to examine the splitter plate effect on flow regimes. The other main objective is to stabilize the critical gap spacing. Furthermore, we want to systematically examine the change over behavior of the mechanism of wake structure behind the downstream cylinder and amplitude variation of fluid forces.

This paper is ordered as follows: The proposed problem, numerical method, initial and boundary conditions are discussed in Section 2. The effect of computational domain and code validation is done in section 3 . The computed results are presented in section 4 . Finally, the conclusion is presented in section 5 .

\section{Problem Description and Numerical Details}

2.1. Problem Description. A schematic representation of the flow through two fixed tandem SCs in presence of attached splitter plate is shown in Figure 1. D, $l$ and $g$ are the width of the cylinder, length of the splitter plate and spacing between the cylinders, respectively. $C_{1}$ and $C_{2}$ are the first and second cylinders, respectively. $L_{u}, L_{d}, L_{x}$, and $L_{y}$ are the upstream location, downstream location, length, and height of the domain, respectively. Parabolic velocity profile is adopted at the entrance position of the domain, while the convective boundary condition is adopted at the exit of the domain [27]. No-slip boundary condition is applied to the surface of the cylinders and splitter plate [28]. Free-slip boundary condition is adopted at the top and bottom lateral boundaries of the channel [29]. The method of momentum exchange [30] is used for the calculation of forces.

2.2. Lattice Boltzmann Method. We have established the 2-D code for the flow through two tandem SCs with and without attached splitter at $(g / D, l / D)=(0-10,0.5-10)$ for $\operatorname{Re}=100$. The lattice Boltzmann method (LBM) is applied for the numerical results obtained in this paper. As compared to well known numerical techniques, the LBM is relatively new technique [28]. It was developed by Frisch et al. [31].

Here we will present a short overview of this method. In this study we applied $Q_{2} N_{9}$ (where $Q$ is the space dimension and $N$ is the number of particles) lattice model. In this model, each computational node consists of a rest particle $(i=0)$ along with eight moving particles $(i=1-8)$ (see Figure 2 ).

The density evolution equation is given by;

$$
g_{i}\left(x+e_{i}, t+1\right)=g_{i}(x, t)-\frac{\left[g_{i}(x, t)-g_{i}^{(0)}(x, t)\right]}{g_{i}(x, t)-\tau},
$$

where, $g_{i}, g_{i}^{(0)}, t, x, e_{i}$ and $\tau$ are the particle distribution function, the corresponding equilibrium distribution function, dimensionless time, position of particles, velocity directions, and single relaxation time, respectively.

The corresponding equilibrium distribution function is:

$$
g_{i}^{(0)}=\rho \omega_{i}\left(1+3\left(e_{i} \cdot u\right)+4.5\left(e_{i} \cdot u\right)^{2}-1.5 u^{2}\right) .
$$

Here, $\rho, u$, and $\omega_{i}$ are the fluid density, velocity and corresponding weighting functions $\left(\omega_{i}=0\right.$ for $i=0, \omega_{i}=1 / 9$ for $i=1-4$, and $\omega_{i}=1 / 36$ for $\left.i=5-8\right)$, respectively. Equation (1) can be solved by two steps: collision which use a Bhatnagar-Gross-Krook (BGK) operator [32, 33] and propagation. These steps can be defined as:

Collision:

$$
g_{i}^{(\text {new) }}(x, t)=g_{i}(x, t)-\frac{\left[g_{i}(x, t)-g_{i}^{(0)}(x, t)\right]}{g_{i}(x, t)-\tau} .
$$

Streaming:

$$
g_{i}\left(x+e_{i}, t+1\right)=g_{i}^{(\text {new })}(x, t) .
$$

Equations (5) and (6) are used to calculate the density and velocity at each computational node 


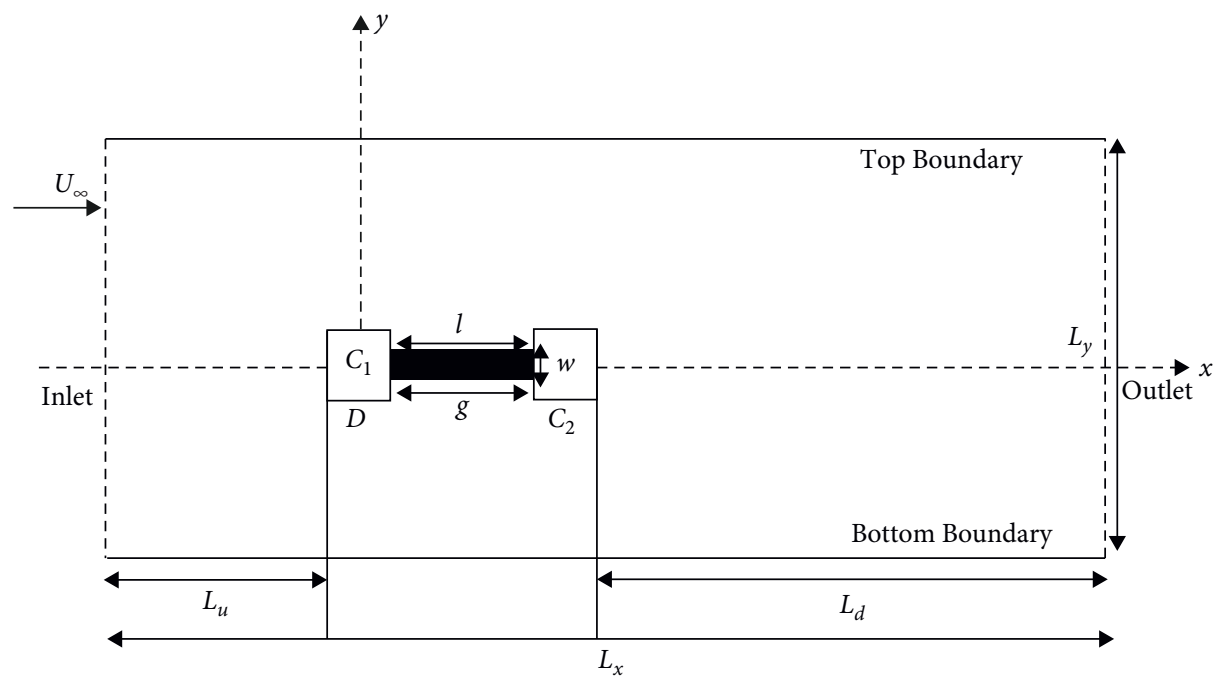

Figure 1: Schematic diagram of flow past two inline square cylinders in confined channel.

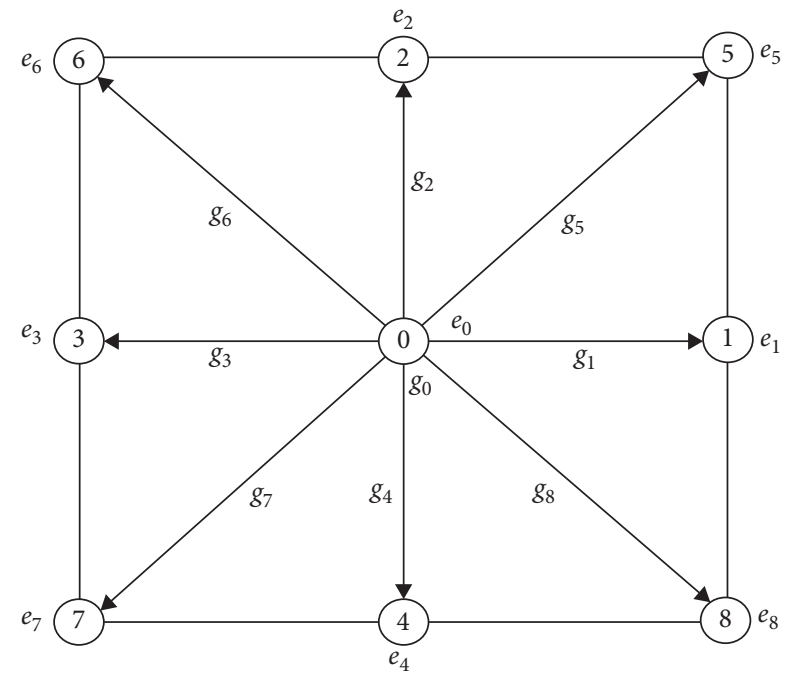

Figure 2: LBM lattice velocities on square structure.

$$
\begin{gathered}
\rho=\sum_{i} g_{i}, \\
\rho u=\sum_{i} g_{i} e_{i} .
\end{gathered}
$$

The boundary conditions are applied after the streaming step (equation (4)), and the entire process is solved iteratively. The pressure can be calculated from the equation of state: $p=\rho c_{s}^{2}$; where $c_{s}$ is the speed of sound and its value is $1 / \sqrt{3}$.

\section{Computational Domain Study and Code Validation}

In this section we will present the grid independence and code validation.
3.1. Grid Independence Study. Table 1 presents the grid independence study. We have taken the error with respect to $D=30$. From the results it is observed that as the size of $D$ is increased from 20 to 30 , the maximum variation in mean drag coefficient $\left(C_{D \text { mean }}\right)$ and Strouhal number ( $S t=f s D / U_{\infty}$, where $f s$ is the vortex shedding frequency) are $0.336 \%$ and $0.9 \%$, respectively. Therefore, for the rest of simulation we used $D=20$.

3.2. Computational Domain. The computational domain size strongly affects characteristics of flow around bluff bodies. The small change in computational domain changes the vortex shedding procedure, which affects the physical parameters. We have calculated the $C_{D \text { mean }}$ and $S t$ for different values of $L_{u}, L_{d}$ and $L_{y}$, so that we can choose a suitable domain. The data in Table 2 shows that there is a slight difference in between the resulted values. So we can consider any case given in Table 2. We will simulate the given problem by using $L_{u}=8 D ; L_{d}=25 D$ and $L_{y}=10 D$. The numbers 1 and 2 in subscripts represents the physical parameters of upstream and downstream cylinders.

3.3. Code Validation. The validation of code is done for the flow past single cylinder for $\mathrm{Re}=100$. For the validation of the code we have used only two parameters that is $C_{D \text { mean }}$ and St. For comparison the numerical results of Dutta et al. [34] as well as experimental data of Norberg et al. [35] and Okajima [36] are given in Table 3. The results shows that the present calculations of $C_{D \text { mean }}$ and Stare in good agreement with the experimental data of Norberg et al. [35] and Okajima [36] and numerical data of Dutta et al. [34].

\section{Results and Discussion}

A two-dimensional (2D) numerical study is carried out to study the effect of $g / D$ and $l / D$ at $\operatorname{Re}=100$. The splitter plate is attached in the middle of the two cylinders. In this study 
Table 1: Cylinder size effect on integral parameters at $\mathrm{Re}=100$.

\begin{tabular}{lccc}
\hline$D$ & 10 & 20 & 30 \\
\hline$C_{D \text { mean }}$ & $1.1916(2.79 \%)$ & $1.1622(0.34 \%)$ & 1.1583 \\
$S t$ & $0.1271(3.78 \%)$ & $0.1235(0.9 \%)$ & 0.1223 \\
\hline
\end{tabular}

TABle 2: Effect of computational domain at $g / D=1$.

\begin{tabular}{lcccccc}
\hline$L_{u}$ & $L_{d}$ & $L_{y}$ & $C_{D \text { mean1 }}$ & $C_{D \text { mean2 }}$ & $S t_{1}$ & $S t_{2}$ \\
\hline $8 D$ & $25 D$ & $10 D$ & 1.0873 & -0.0828 & 0.1018 & 0.1018 \\
& & & $(3.08 \%)$ & $(3.8 \%)$ & $(3.41 \%)$ & $(3.41 \%)$ \\
$8 D$ & $40 D$ & $10 D$ & 1.1208 & -0.0796 & 0.1054 & 0.1054 \\
$12 D$ & $25 D$ & $10 D$ & 1.1144 & -0.0860 & 0.0987 & 0.0989 \\
& & & $(2.43 \%)$ & $(3.86 \%)$ & $(3.05 \%)$ & $(3.04 \%)$ \\
$8 D$ & $25 D$ & \multirow{2}{*}{$10 D$} & 1.0873 & -0.0828 & 0.1018 & 0.1018 \\
& & & $(3.53 \%)$ & $(2.90 \%)$ & $(3.59 \%)$ & $(3.60 \%)$ \\
$8 D$ & $25 D$ & $14 D$ & 1.0489 & -0.0 .852 & 0.1056 & 0.1058 \\
\hline
\end{tabular}

TABLE 3: Comparison of present and previous results.

\begin{tabular}{lcccc}
\hline & Present & $\begin{array}{c}\text { Dutta } \\
\text { et al. [34] }\end{array}$ & $\begin{array}{c}\text { Norberg } \\
\text { et al. [35] }\end{array}$ & Okajima [36] \\
\hline$C_{\text {Dmean }}$ & 1.162 & 1.15 & 1.44 & 1.6 \\
St & 0.124 & 0.13 & 0.145 & 0.14 \\
\hline
\end{tabular}

we will discuss the vorticity contours visualization, time analysis of $C_{D}$ and $C_{L}$ and power spectra of $C_{L}$. During this study the $g / D$ will be varied from 0 to 10 and $l / D$ will be varied from 0.5 to 10 .

4.1. The Flow Over Two Tandem Square Cylinders without Splitter Plate. Figures 3(a)-3(f) presents the instantaneous flow visualization of flow field through two SCs in tandem arrangement without splitter plate. The different $g / D$ values in Figures 3(a)-3(f) represented the flow filed characteristics is $\mathrm{SBB}(g / D=0.5$ and 1$)$, SF regime $(g / D=2$ and 3$)$, FDF regime $(g / D=5)$ and FDTRVS regime $(g / D=9)$, respectively. The pattern of vortex was a single row in SBB flow regime (Figure 3(a)). The phenomenon was analogous to the flow past a single SC. When the value of $g / D$ is increased up to 1 , the shear layers detached from the $C_{1}$ reattach on the upper surface of the $C_{2}$ (Figure $3(\mathrm{~b})$ ). When the $g / D$ is increased to 2 and 3 , no vortex shedding occurs behind the second cylinder (Figures 3(c), and 3(d)). When $g / D=5$, the fully developed vortex shedding can be clearly seen behind the upstream square cylinder. Under these conditions, the flow behind the downstream SC was almost matching to that around single cylinder, and there was a little affect by the upstream SC (Figure 3(e)). On the other hand, the downstream SC was still affected by the upstream SC, appearing to be a two-rows vortex street at $g / D=9$ (Figure 3(f)).

In the FDF regime, the two inline cylinders separately shed vortices at the same frequency. Igarashi [37] also reported that the two inline circular cylinders shed vortices at the same frequency at reasonably large spacing ratio. It is observed that the FDF or co shedding flow regime occurs at $g / D=5$ where the vortices shed separately from the two cylinders but with same frequency. The negative vortex shedding from $C_{1}$ hits the front face of the $C_{2}$ and at the same time the negative and positive shed vortices develop from the cylinder $C_{2}$. These different shed vortices interact with each other and results in a FDTRVS regime with multi-frequency variation in the spectra of the lift coefficient of $C_{2}$ (ref. spectrum in Figure 4(j)).

Instantaneous streamline visualization shows the alternate generation of negative and positive shed vortices presented in Figures 5(a)-5(f). It is found that the wake width and vortex formation length are considerably dependent on spacing ratio. In SBB regime, the shear layers separated from the front edges of $C_{1}$ quickly reattached on the lateral surface of $C_{2}$, forming two small recirculation regions within the cylinders (Figure 5(b)). Due to strong suction a negative drag value is observed for $C_{2}$. At $g / D=5$, both the cylinders $\left(C_{1}\right.$ and $\left.C_{2}\right)$ shed vortices and the flow characteristics is called a FDF regime.

Time variation $C_{D}$ and $C_{L}$ of both cylinders are shown in Figures 6(a)-6(l) for different spacing ratios. As spacing ratio increases, the amplitude of drag coefficient also increases for FDF regime and FDTRVS regime (Figures 6(i), and 6(k)). It is observed that the $C_{D}$ of $C_{2}$ is modulated. At $g / D=0.5$ and 1 , the $C_{L}$ become sinusoidal, and the first cylinder and second cylinder shed vortices result in an inphase mode (Figures 6(b), and 6(d)). At $g / D=2$, the $C_{L}$ of both cylinders becomes constant (Figure 6(f)).

The spectra graph shows a single dominant peak corresponding to the primary frequency; no secondary frequency was observed in the spectra in case of single bluff body flow regime (Figures 4(a)-4(d)). The small peak in the power spectra (Figures $4(\mathrm{~h})$ and $4(\mathrm{j})$ ) suggests that the second cylinder flow is still affected by the first cylinder.

4.2. The Flow Around Two Tandem Square Cylinders with Attached Splitter Plate. Figures 7(a)-7(e) presents the vorticity contours visualization of flow field around through inline SCs. The different splitter plate length within the spacing between the two cylinders represented the flow filed characteristics in reattachment flow regime $(g / D=l / D=0.5$ and 1) and steady flow regime $(g / D=l / D=2.5,5$ and 10$)$. In shear layers reattachment flow (SLR) regime, the shear layers separated from the $C_{1}$ reattach on the upper surface of the $C_{2}$ (Figures $7(\mathrm{a})$ and $7(\mathrm{~b})$ ). When $g / D=l / D \geq 2.5$, the steady flow regime can be clearly seen behind both cylinders (Figures 7(c)-7(e)). Under these circumstances, the flow behind the upstream and downstream SCs was almost similar, and it was hardly affected by the downstream SC. Inspection of Figures 8(a)-8(e) further confirms that the flow through the downstream SC becomes steady only in existence of the splitter plate of $l / D \geq 2.5$.

The higher upward mean lift force is observed for the downstream SC when the two cylinders are closely spaced as the viscous effect is more towards the lower face of the downstream square cylinder. From Figures 9(a), 9(c), 9(e) and $9(\mathrm{~g})$ it can be seen that at $l / D=0.5,1,2.5$ and 10 the $C_{D 1}$ and $C_{D 2}$ are both constants. Furthermore, the $C_{D 2}$ having negative drag coefficient at $l / D=0.5,1$ and 2.5. On the other hand, the lift force has periodic behaviour with decreasing amplitude as the value of $l / D$ increased. Furthermore, at $l /$ 


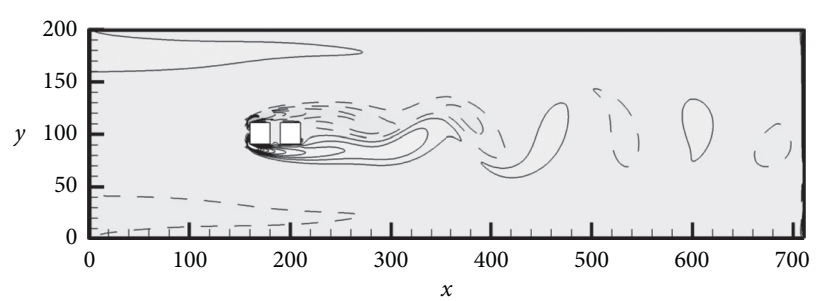

(a)

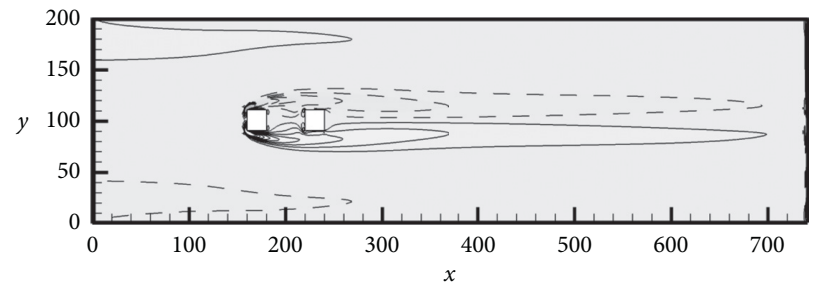

(c)

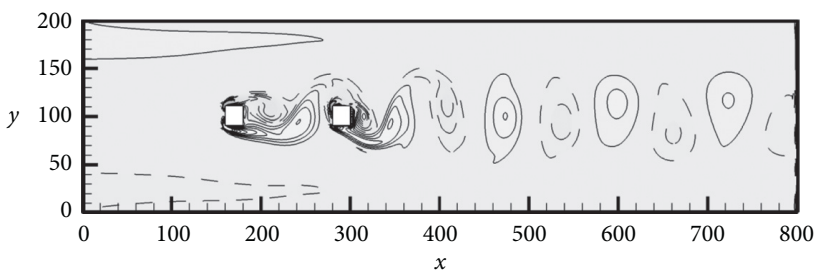

(e)

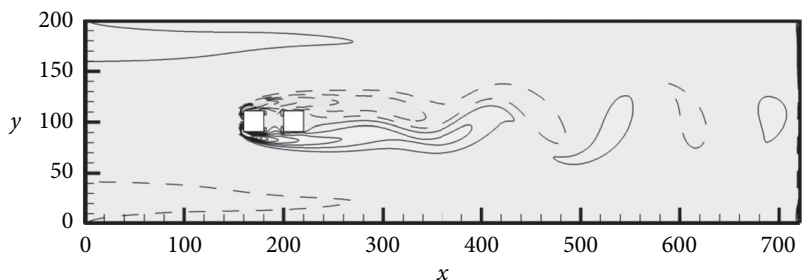

(b)

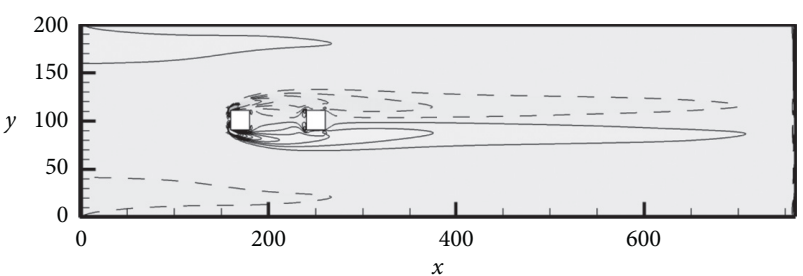

(d)

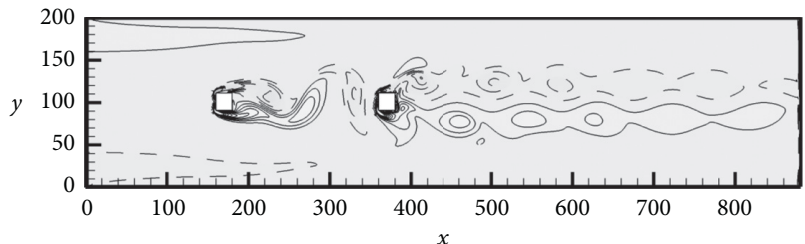

(f)

FIgURE 3: (a-f). Instantaneous vorticity contours visualization at different spacing ratios.

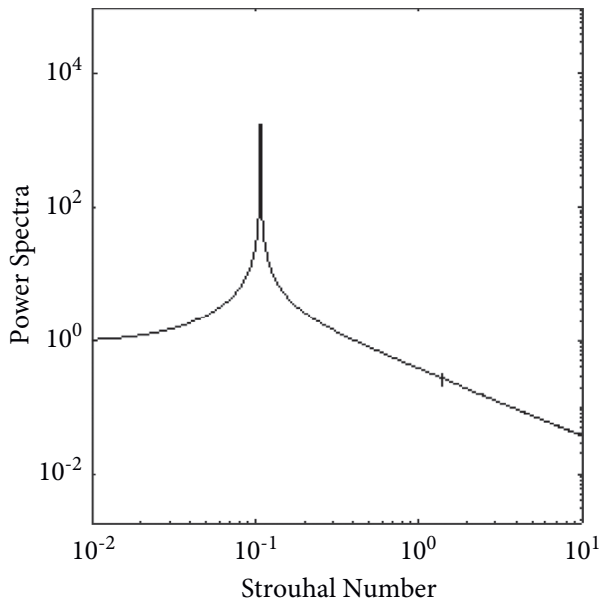

$-S t 1$

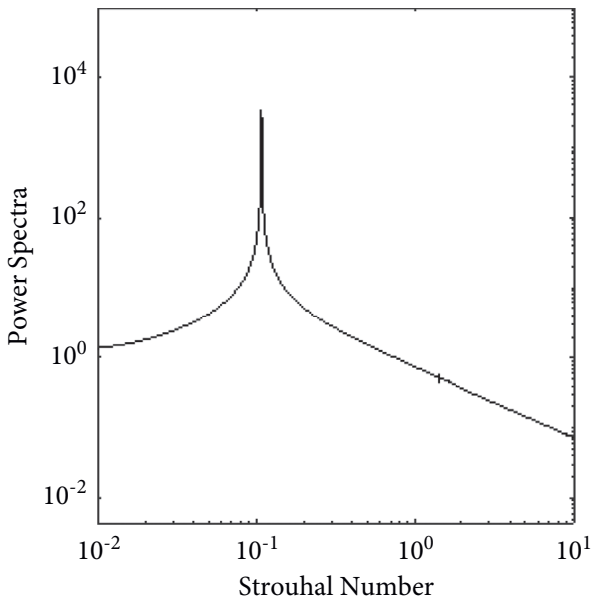

$-S t 2$

(a)

(b)

Figure 4: Continued. 


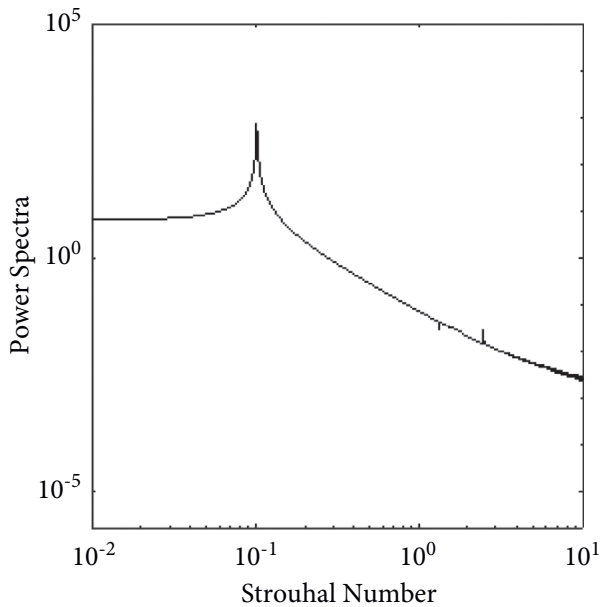

- St 1

(c)

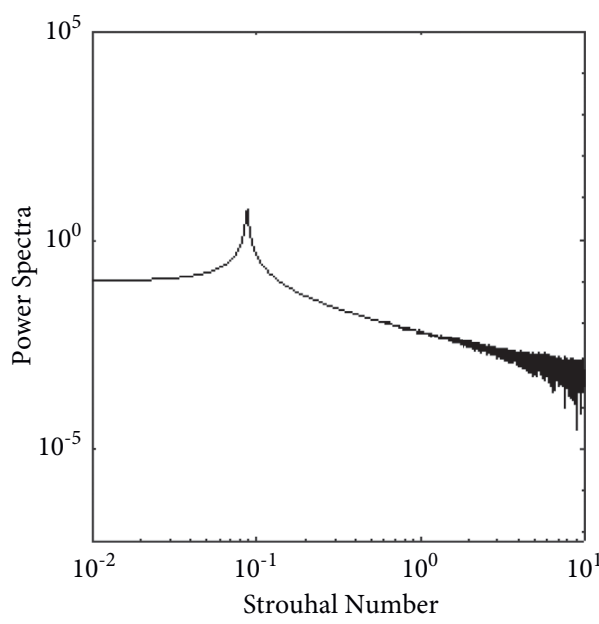

$-S t 1$

(e)

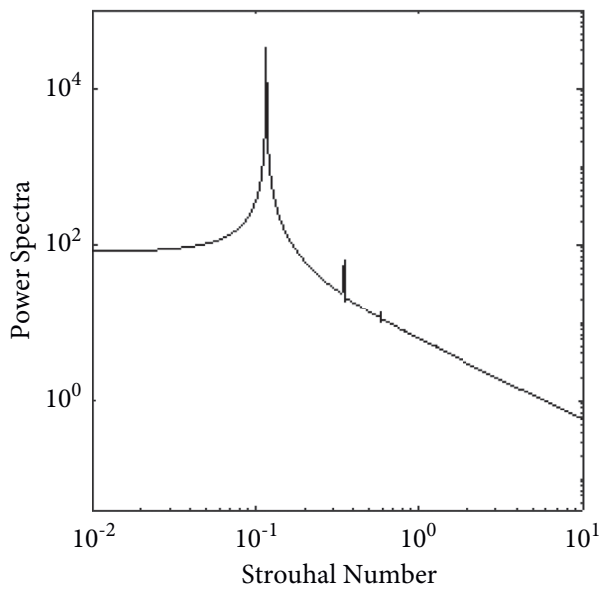

- St 1

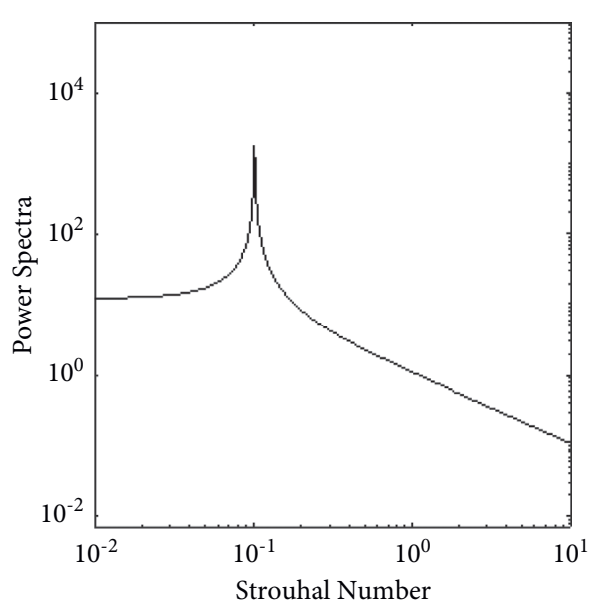

- St2

(d)

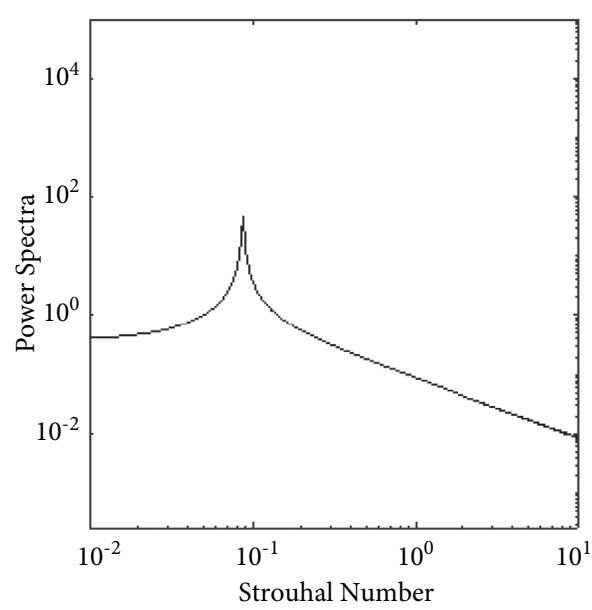

- St2

(f)

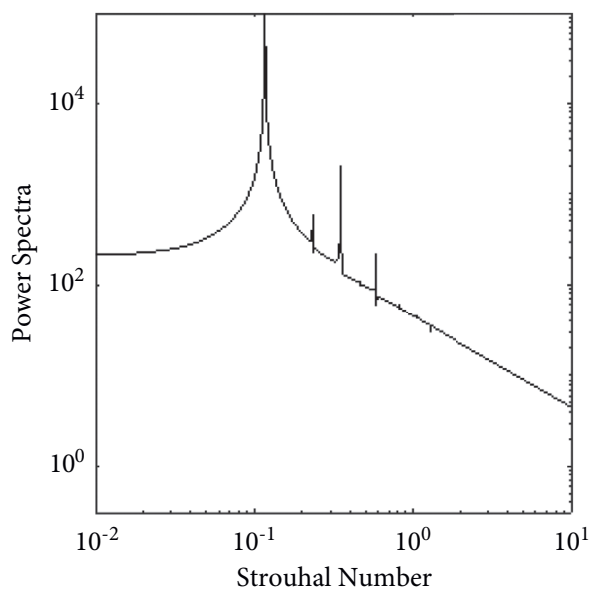

St2

(g)

(h)

Figure 4: Continued. 


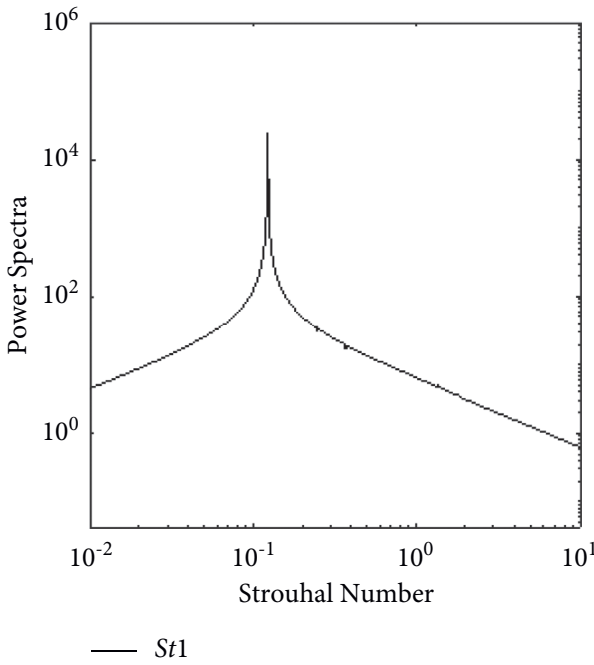

(i)

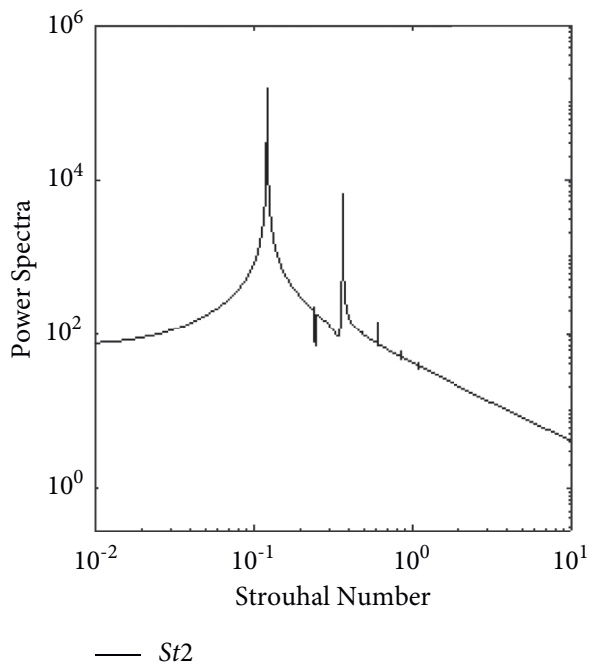

(j)

Figure 4: (a-j). Spectra analysis of lift coefficients at different spacing ratios.

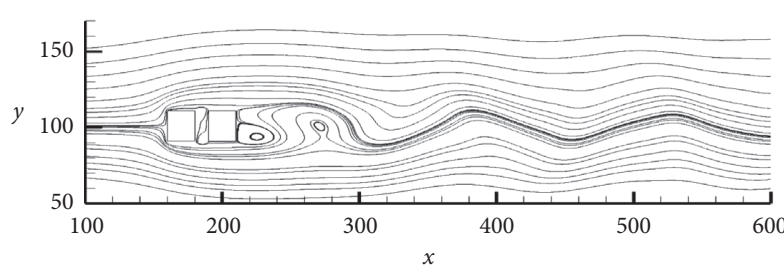

(a)

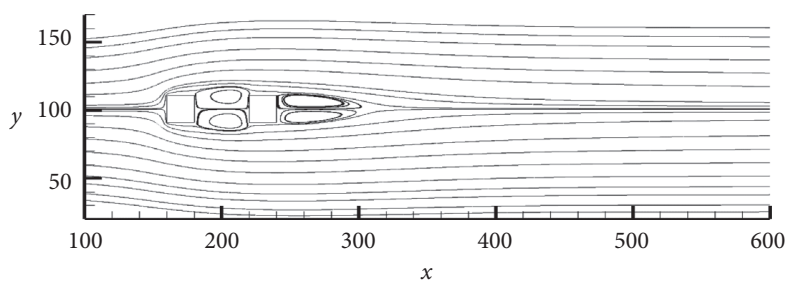

(c)

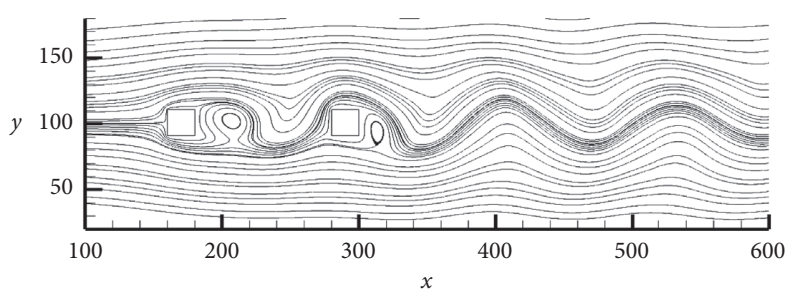

(e)

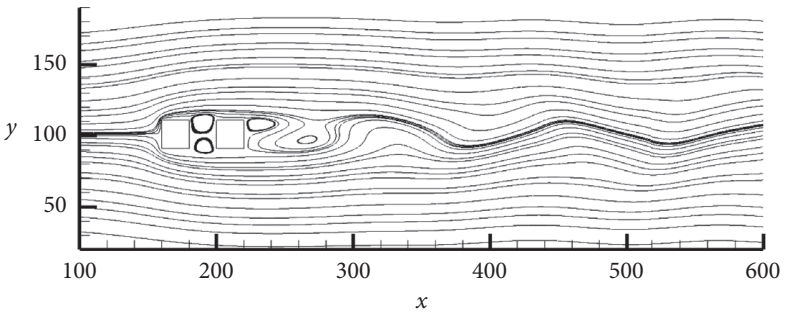

(b)

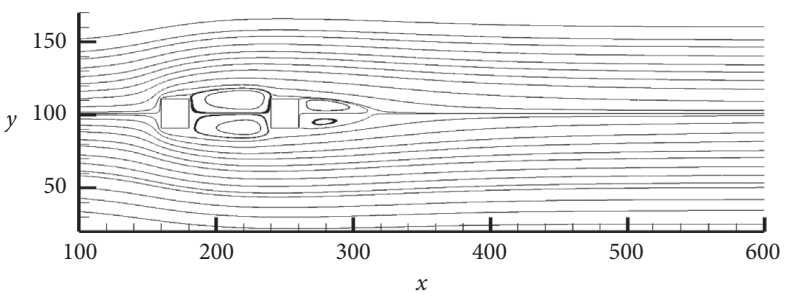

(d)

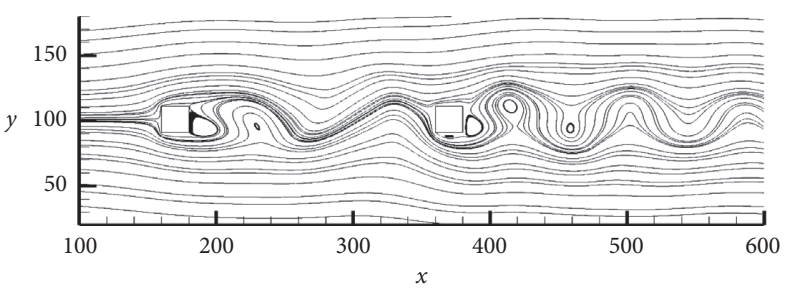

(f)

Figure 5: (a-f). Streamlines visualization at different spacing ratios.

$D=10$, the lift force is a straight line due to steady flow behaviour behind the cylinders.

The power spectra of $C_{L}$ of the two cylinders with different splitter plate length are shown in Figures 10(a)-10(f). At $l / D=0.5$, the lift force oscillation of the upstream cylinder was stable, which ensures that the interference is weak between the cylinders. When the splitter plate length is varied from 0.5 to 1 , the power spectra not changed. With the further increase in splitter plate length, the interference among the two tandem cylinders slowly disappeared, and the oscillation became stable again. For the downstream cylinder, its lift force oscillation had almost the same behaviour as the upstream square cylinder. The difference was observed when the splitter plate length was varying from 5 to 10 . Some 

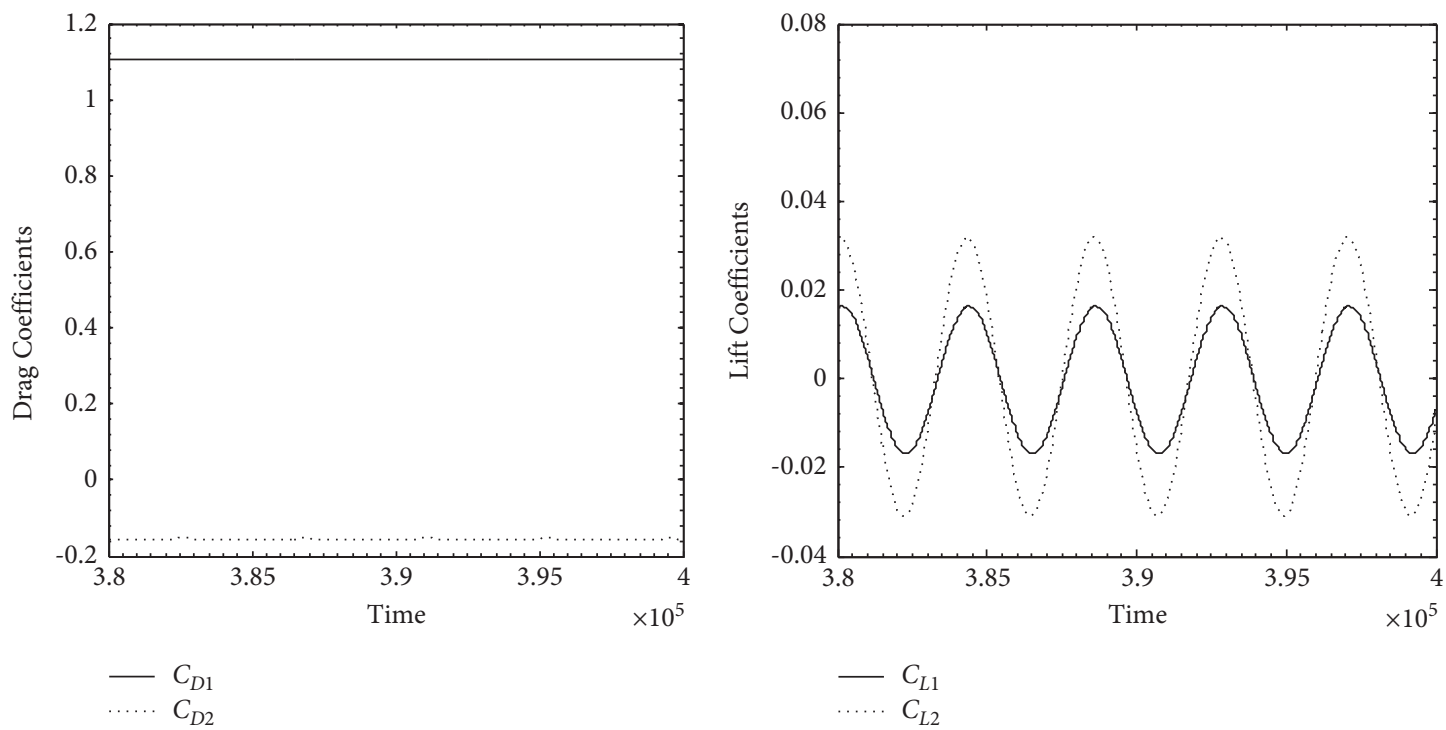

(a)

(b)

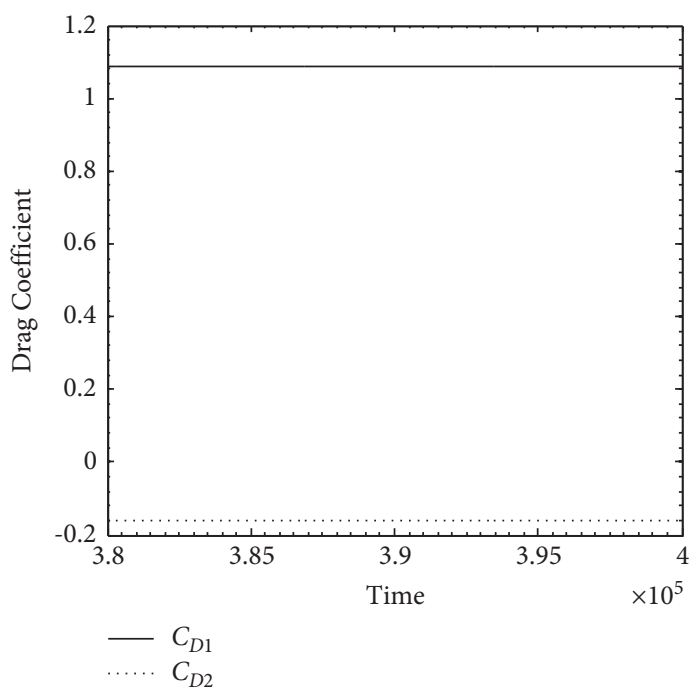

(c)

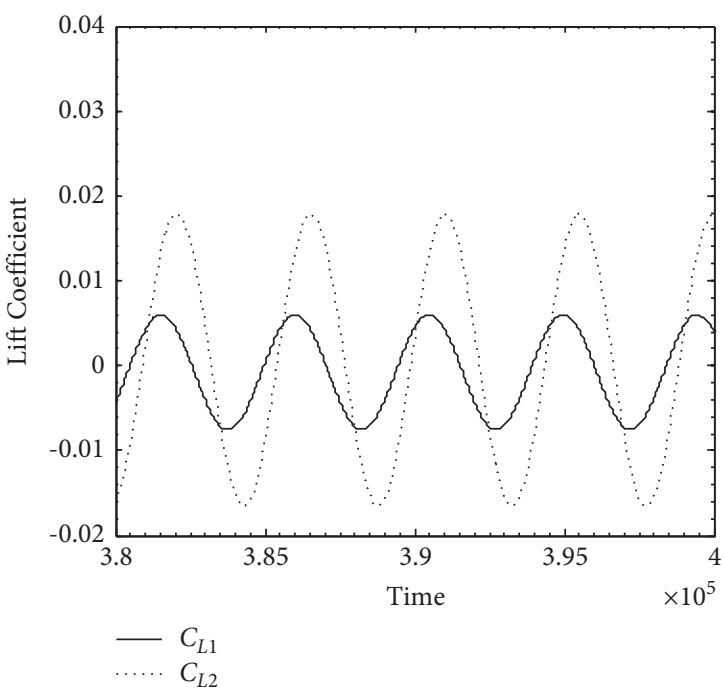

(d)
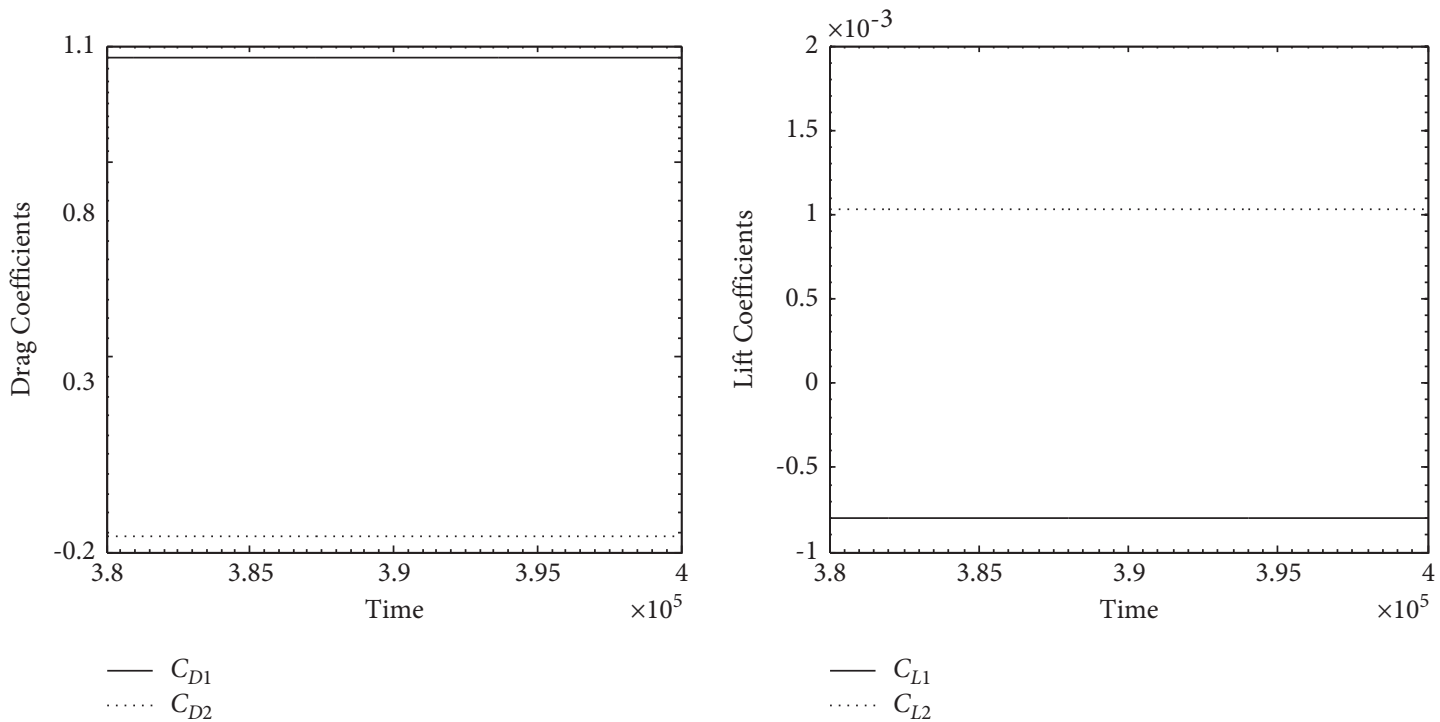

(e)

Figure 6: Continued. 

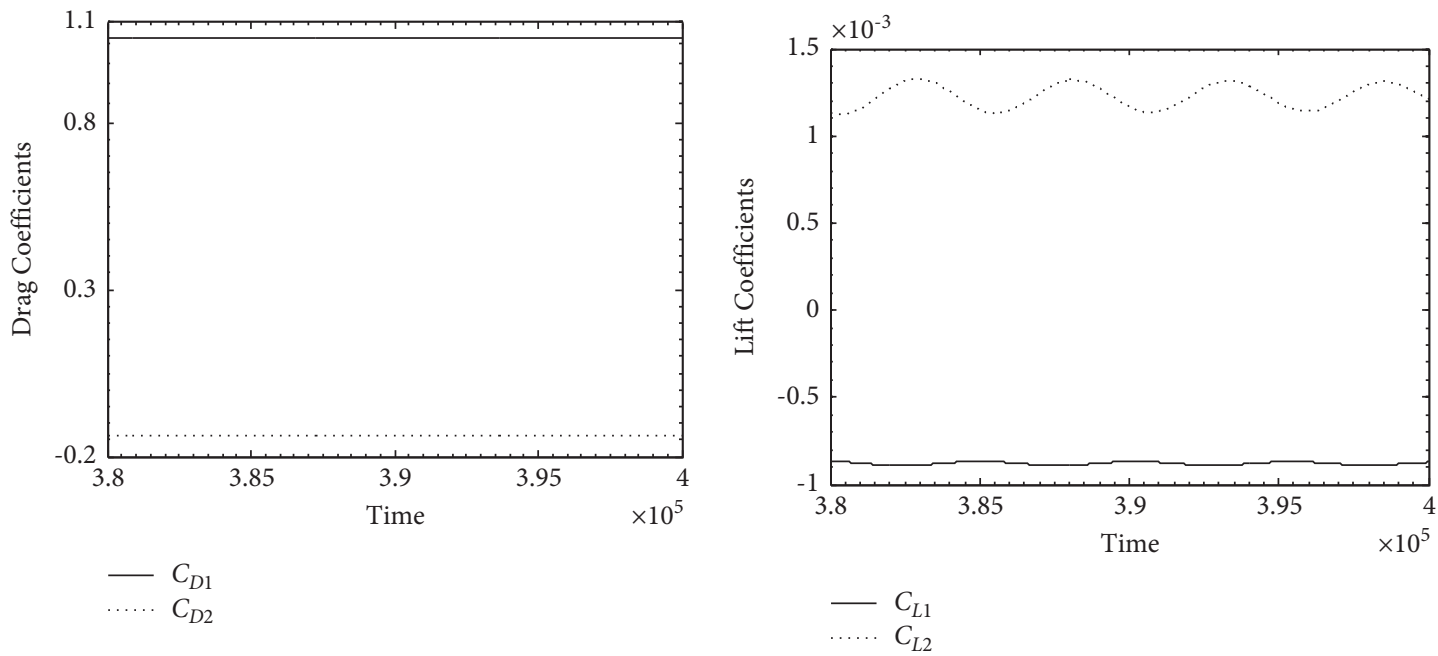

(g)
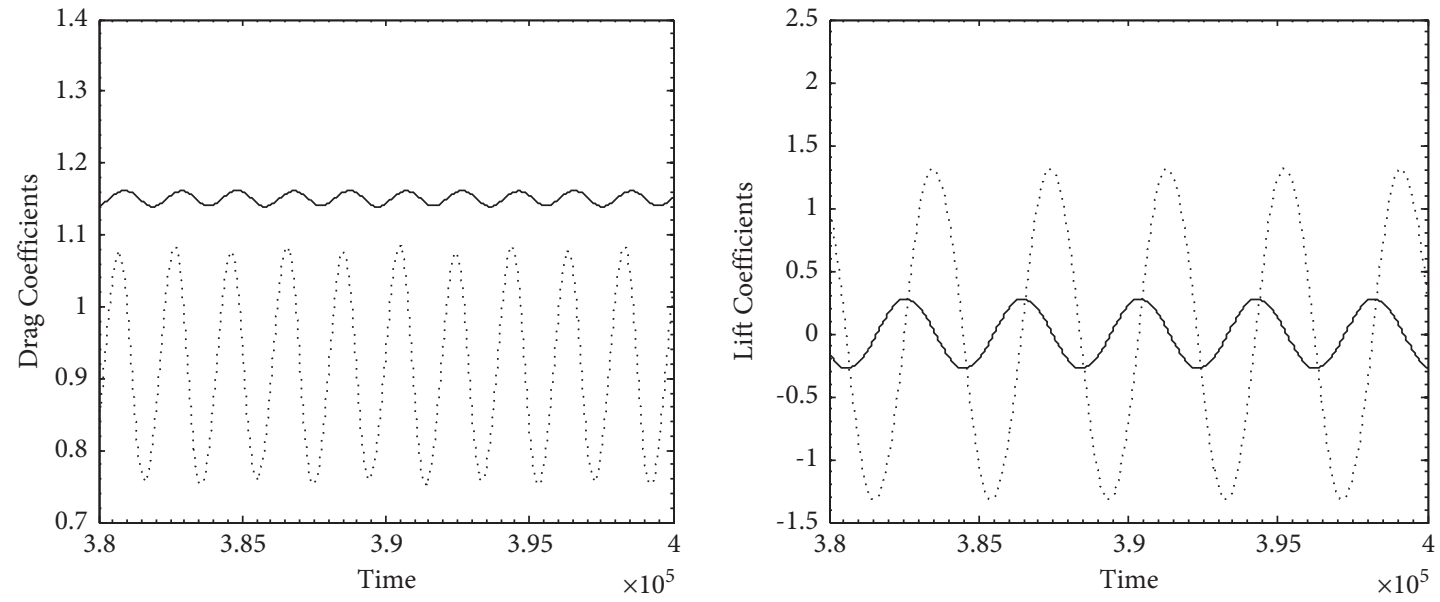

$\begin{array}{ll}- & C_{D 1} \\ \cdots \cdots & C_{D 2}\end{array}$

$\begin{array}{ll}- & C_{L 1} \\ \cdots & C_{L 2}\end{array}$

(i)

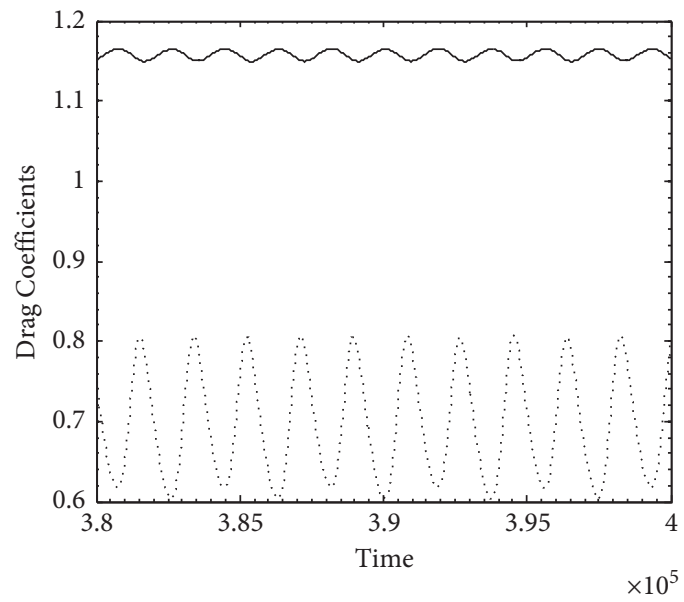

$\begin{array}{ll}- & C_{D 1} \\ \cdots \cdots & C_{D 2}\end{array}$

$\times 10^{5}$

(k)

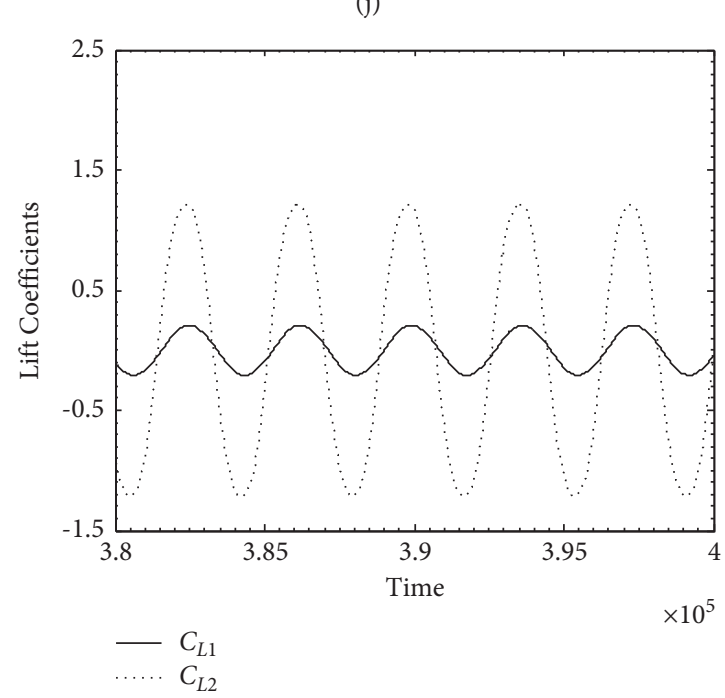

(1)

Figure 6: (a-l). Time variation of $C_{D}$ and $C_{L}$ at different spacing ratios. 


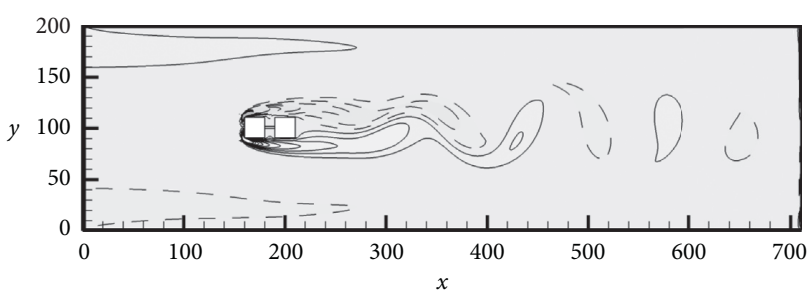

(a)

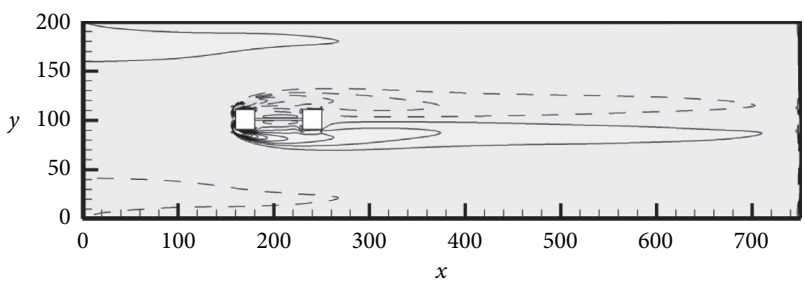

(c)

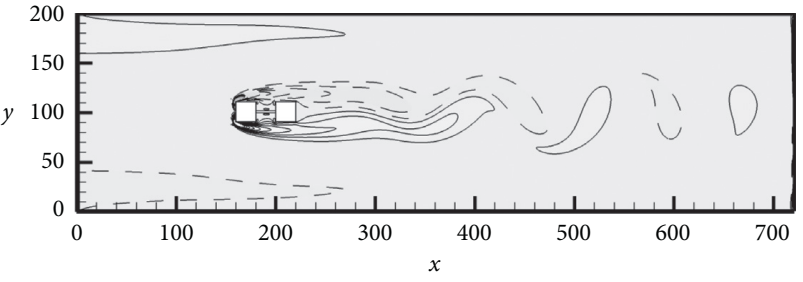

(b)

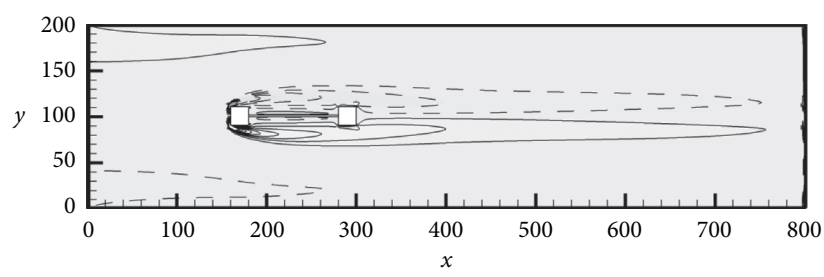

(d)

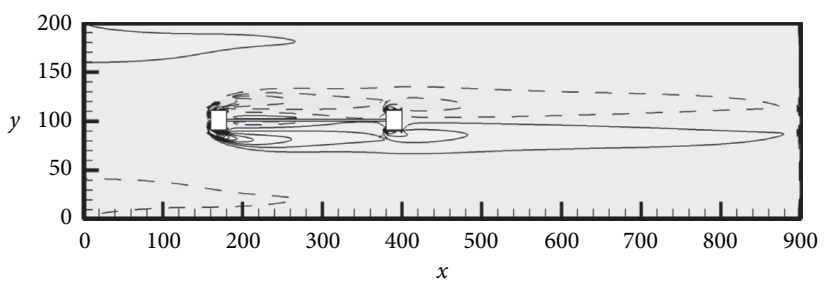

(e)

Figure 7: (a-e). Vorticity contours at different splitter plate lengths

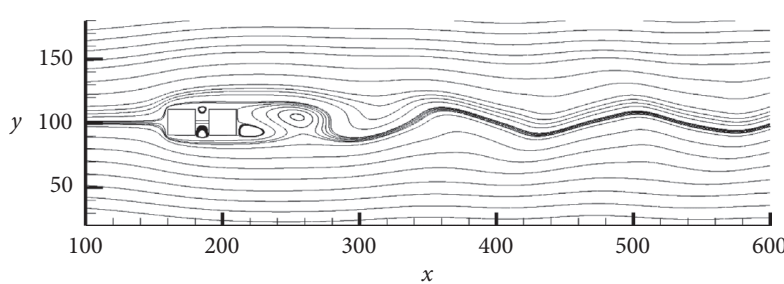

(a)

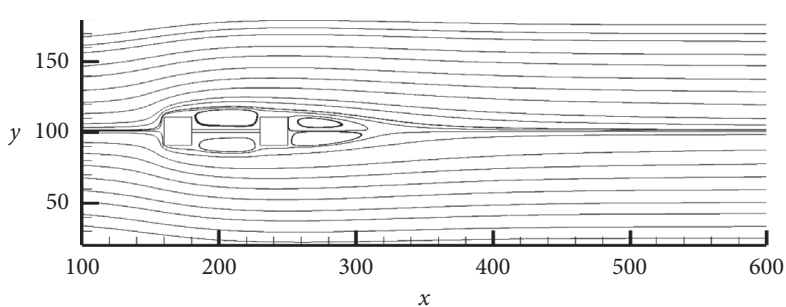

(c)

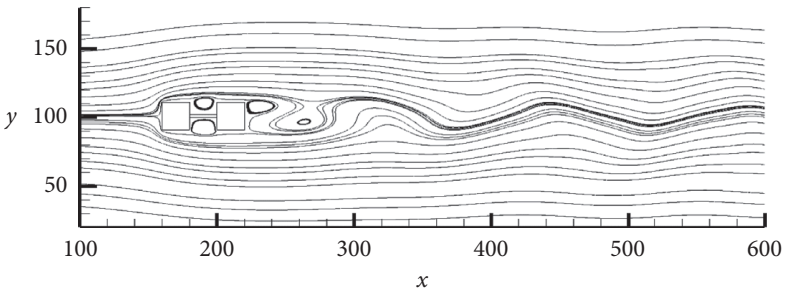

(b)

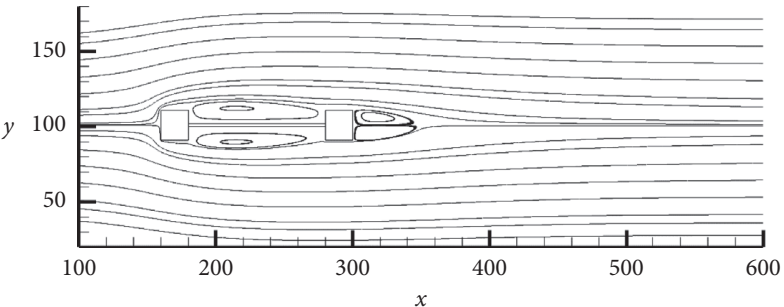

(d)

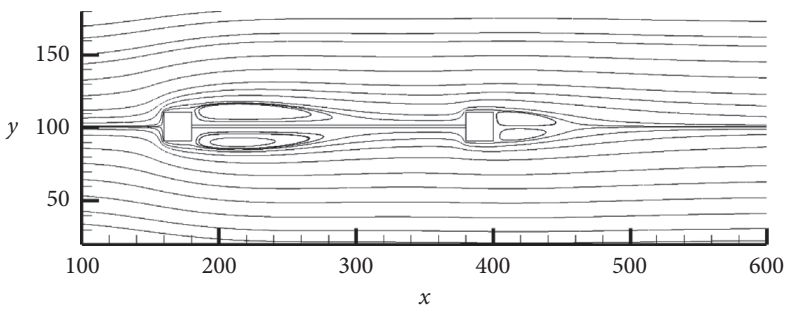

(e)

FIGURE 8: (a-e). Streamlines visualization at different values of splitter plate length. 

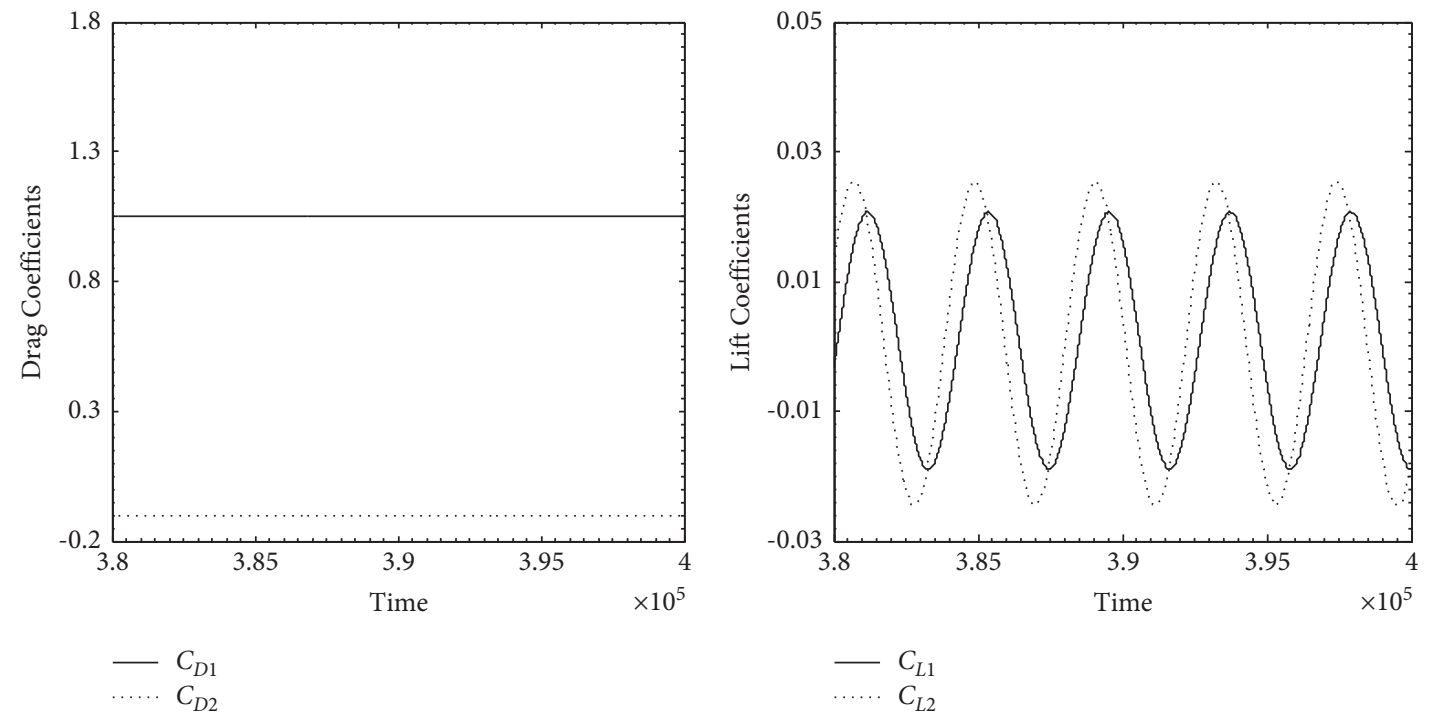

(a)

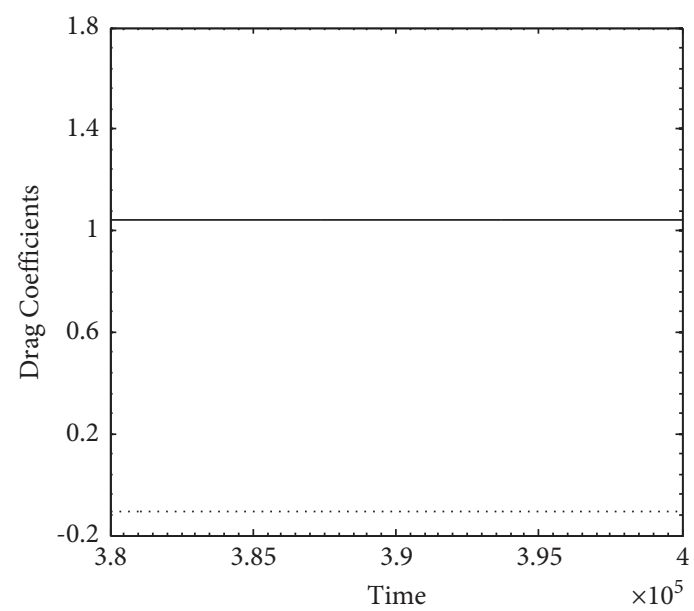

(b)

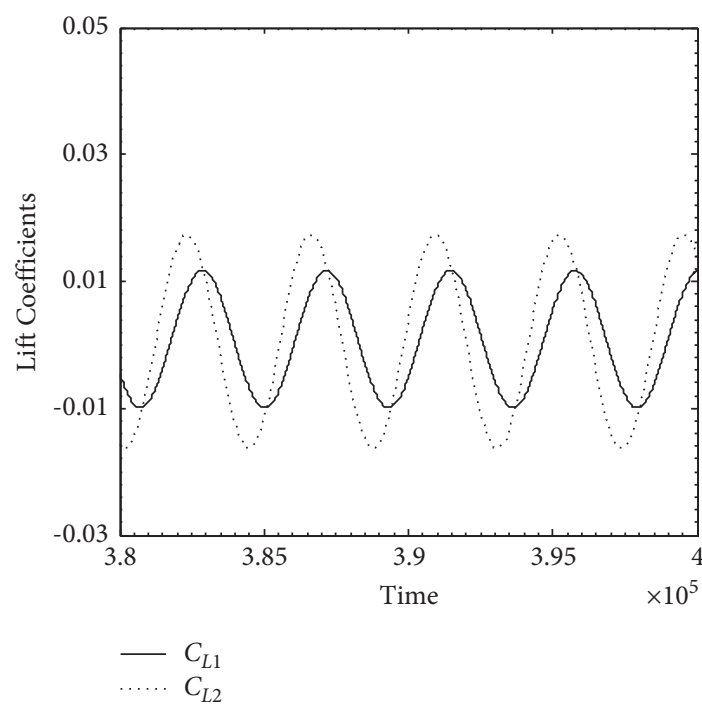

(c)

(d)
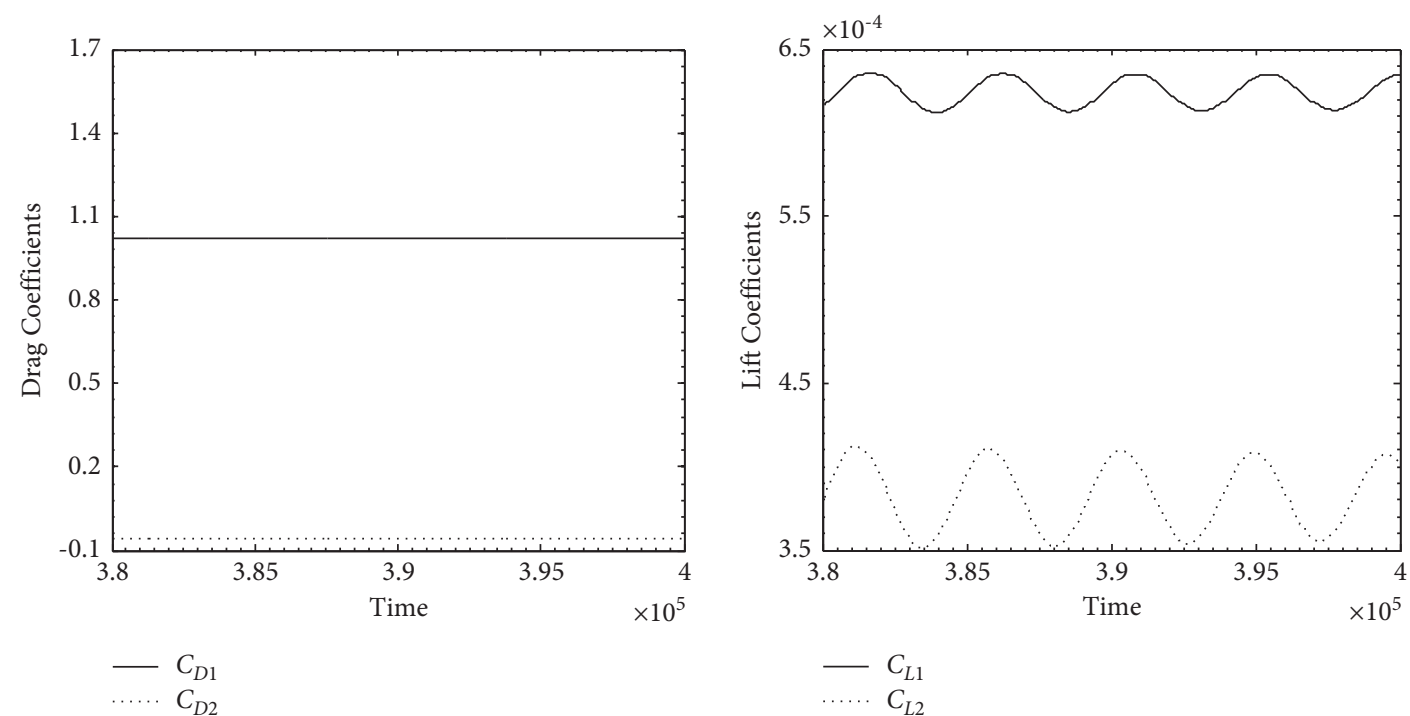

(e)

(f)

Figure 9: Continued. 


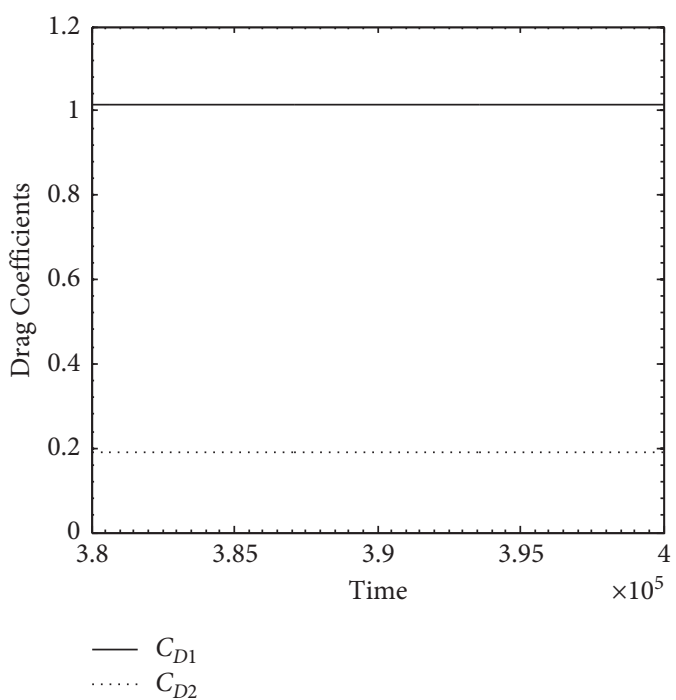

(g)

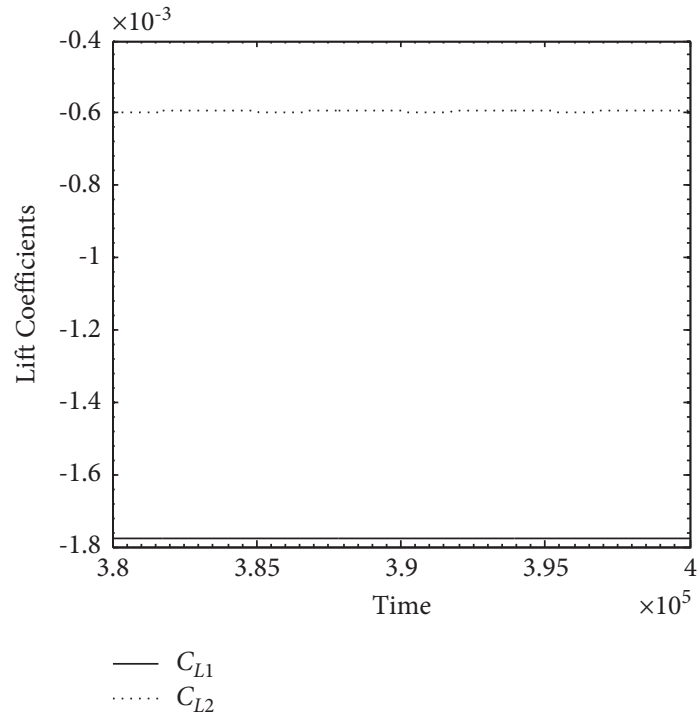

(h)

Figure 9: $(\mathrm{a}-\mathrm{h})$. Time variation of $C_{D}$ and $C_{L}$ at different $l / D$ values.

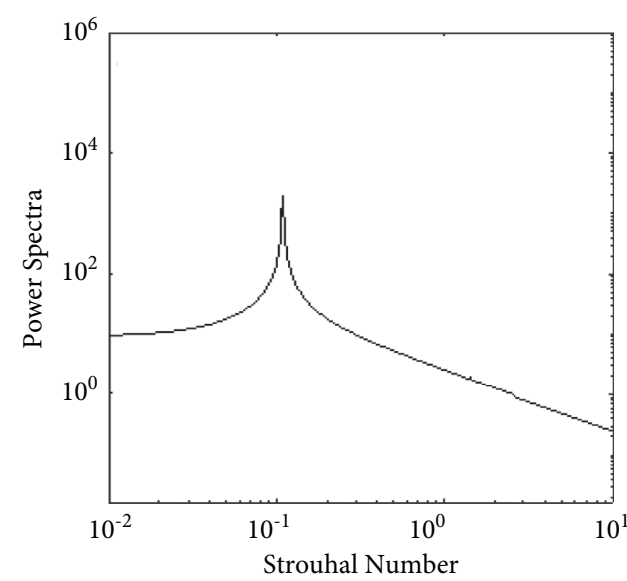

(a)

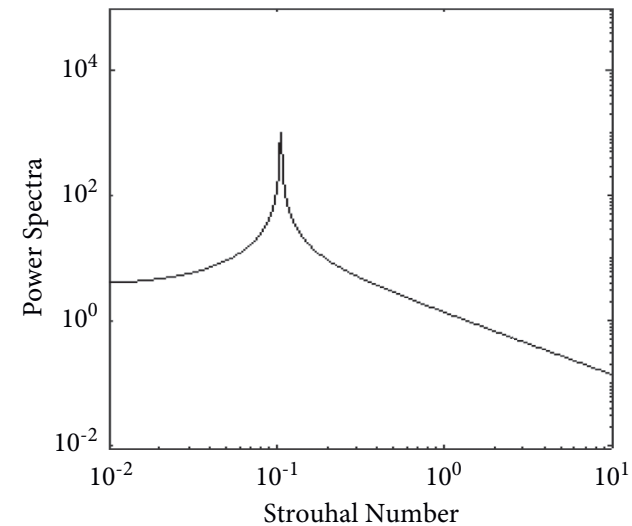

$-S t 1$

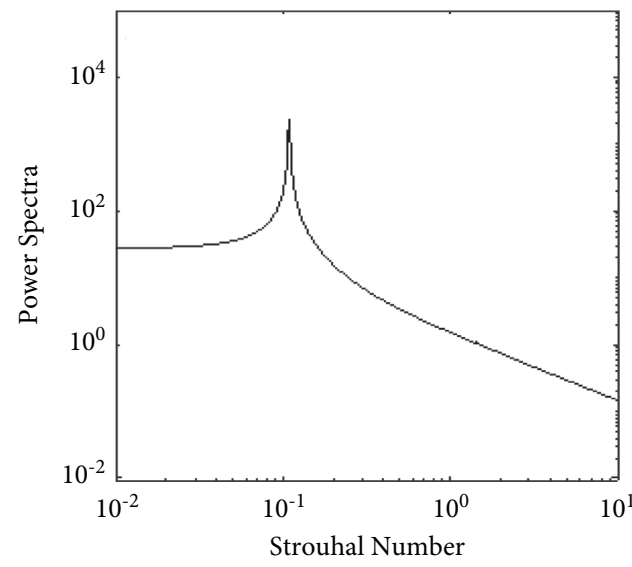

(b)

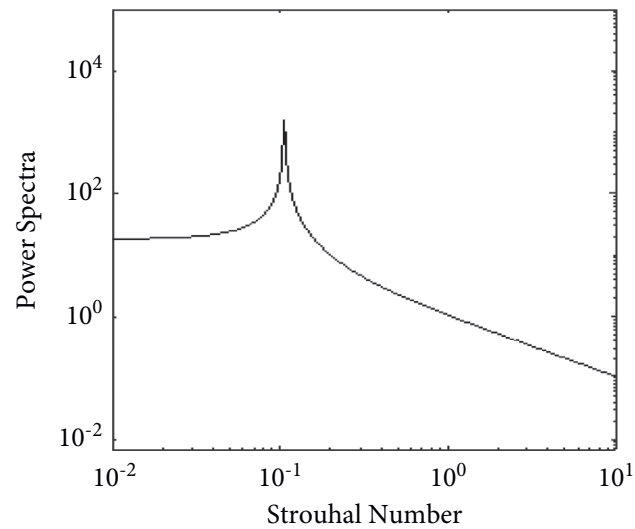

St2

(c)

(d)

Figure 10: Continued. 


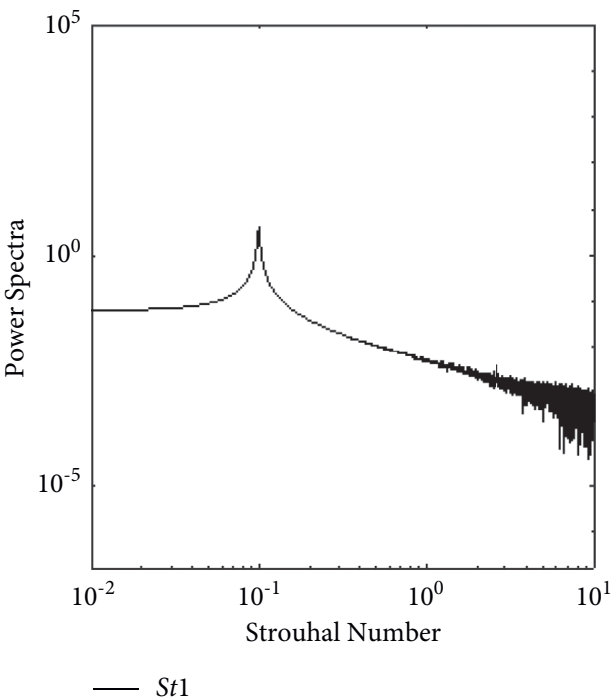

(e)

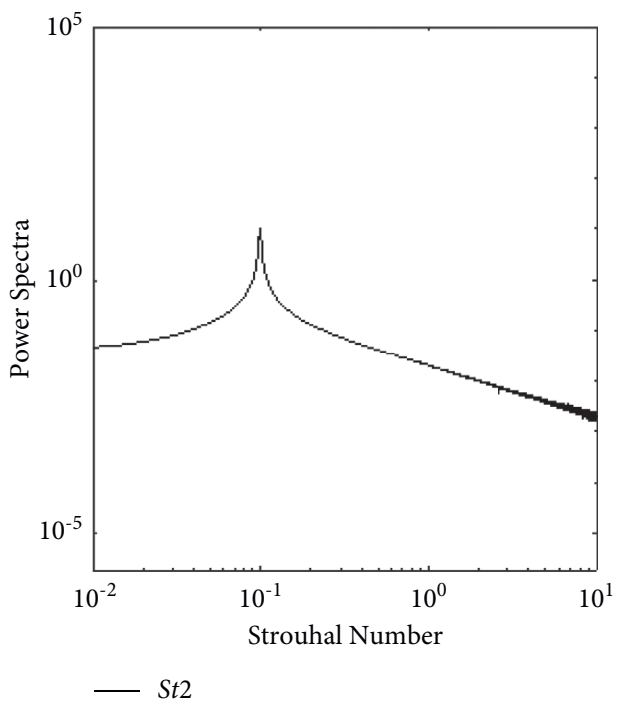

(f)

FIgUre 10: (a-f). The spectra analysis of lift coefficients at different $l / D$ values.

minor peaks in the spectra of the upstream square cylinder were observed. This is due to the vortex generated by the $C_{1}$ directly acts on the $C_{2}$.

The graphical representation of the different observed flow regimes is given in Figure 11. It can be observed that at small value of $g / D$ and splitter plate length the single bluff body flow regime occurs. At intermediate values of $g / D$ and $l / D$ the shear layers reattachment flow regime occurs. The FDF regime and FDTRVS regime can be seen only for the two tandem square cylinders without splitter plate. It can also be observed from the flow diagram that the splitter plate considerably suppressed the vortex shedding behind the cylinders and completely convert it to steady flow regime.

4.3. Analysis of Force Statistics. In Figure 12(a), compared with the $C_{D \text { mean }}$ of a single $S C$, the $C_{D \text { mean }}$ of $C_{1}$ was slightly reduced up to $g / D=4.5$ without splitter plate. As the $g / D$ increased, the $C_{D \text { mean }}$ of the $C_{1}$ remained unchanged and was almost equal to an isolated cylinder. The $C_{D \text { mean }}$ of the $C_{2}$ was considerably smaller than that of single cylinder due to the influence by the wake of the upstream SC. The value of $C_{D \text { mean }}$ of the $C_{2}$ was negative when $g / D$ is varied from 0.5 to 4. When the value of $g / D$ increased to 4 , the $C_{D \text { mean }}$ of $C_{2}$ changed from negative to positive. As the $g / D$ continued to increase $(g / D>4)$, the $C_{D \text { mean }}$ of the downstream cylinder first increased and then slowly starts to decrease. The transition from shear layers reattachment flow regime to two-row vortex street flow regime was actually a positive-negative conversion of the $C_{D 2}$. It is found that in presence with the splitter plate, the value of $C_{D \text { mean }}$ of the $C_{2}$ is considerably smaller than in the cases without splitter plate. The splitter plate length has marginal role on drag reduction for $g / D>5$.

$C_{D r m s 1}$ and $C_{D r m s 2}$ are the root-mean-square drag values of the first cylinder and second cylinder, respectively (Figure 12(b)). The discontinuity is observed in $C_{D \text { rms2 }}$

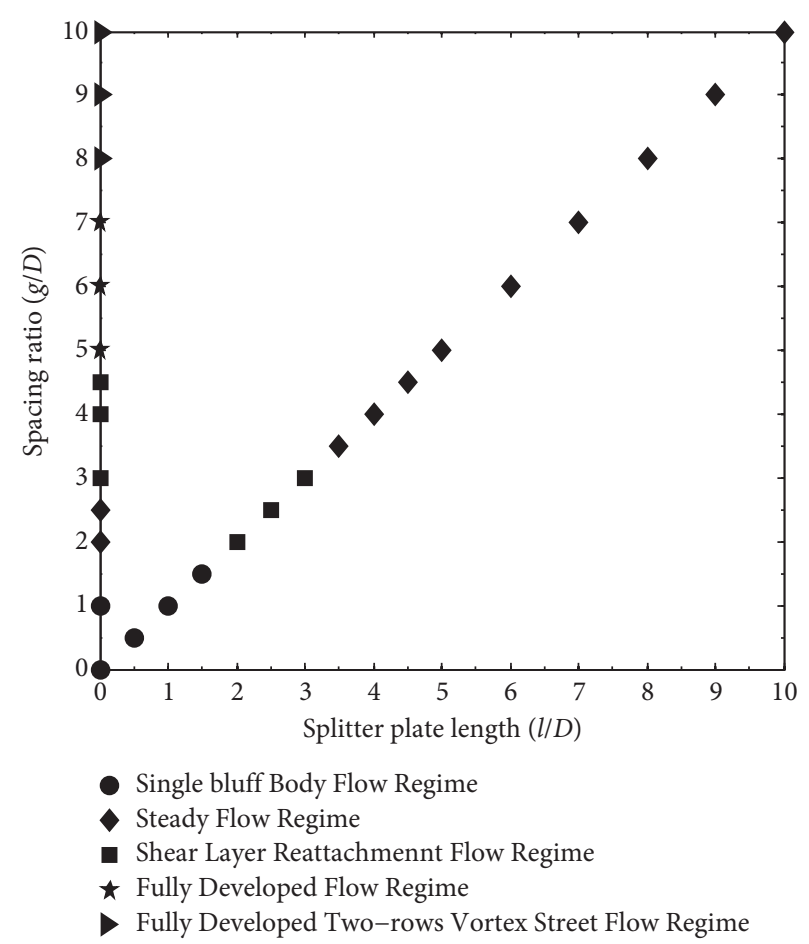

FIGURE 11: Flow regimes observed at different splitter plate lengths and spacing ratio.

without splitter plate in Figure 12(b) for the case of $g / D=4.5$ due to transition of flow regime from shear layers reattachment flow regime $(g / D=4)$ to fully developed flow regime $(g / D=4.5)$. For a splitter plate length of $l / D 1$ to 10 , the $C_{D \text { rms }}$ of both cylinders is nearly equal to that of the isolated cylinder. However, it is found without splitter plate that the $C_{D \text { rms2 }}$ is irregular.

From Figure 13(a), it is seen that the $C_{L}$ of the cylinder $C_{2}$ is more affected as compared to the cylinder $C_{1}$ due to the 


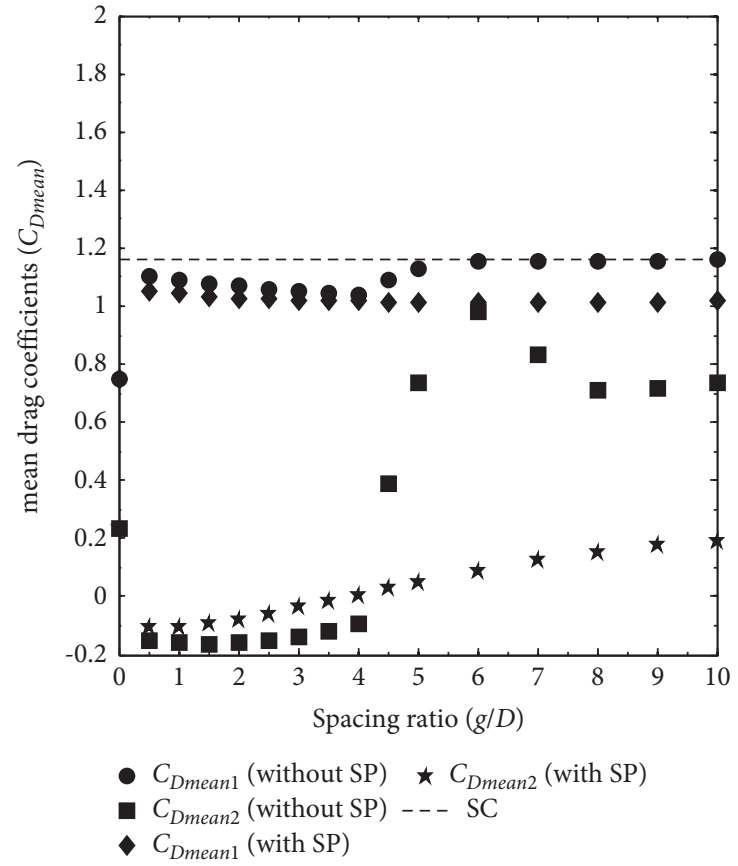

(a)

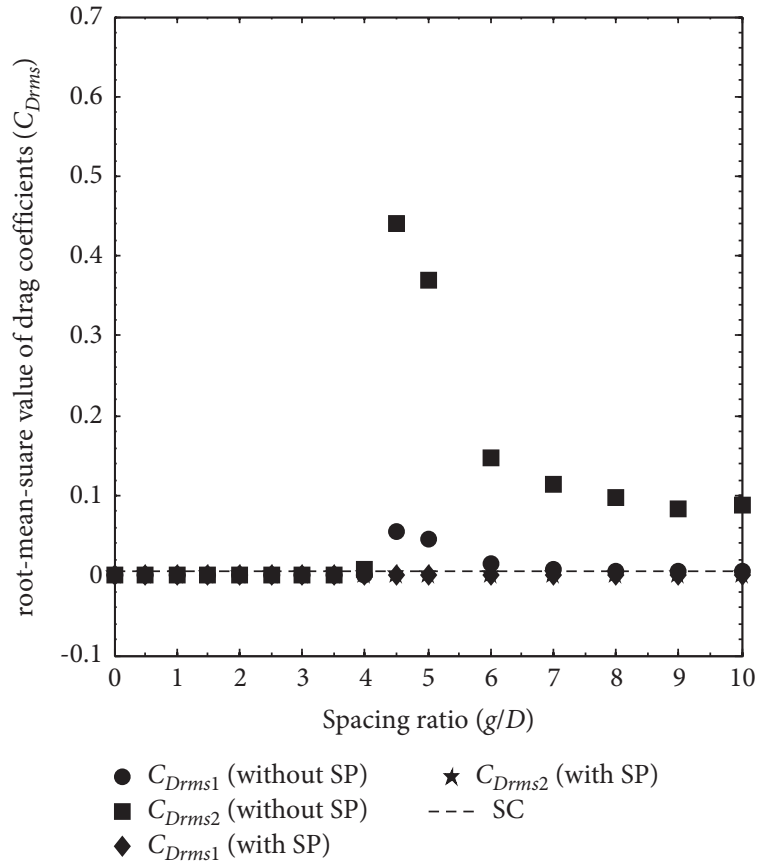

(b)

Figure 12: $(\mathrm{a}, \mathrm{b})$. Variation of $C_{D \text { mean }}$ and $C_{D \text { rms }}$ as a function of spacing ratio.

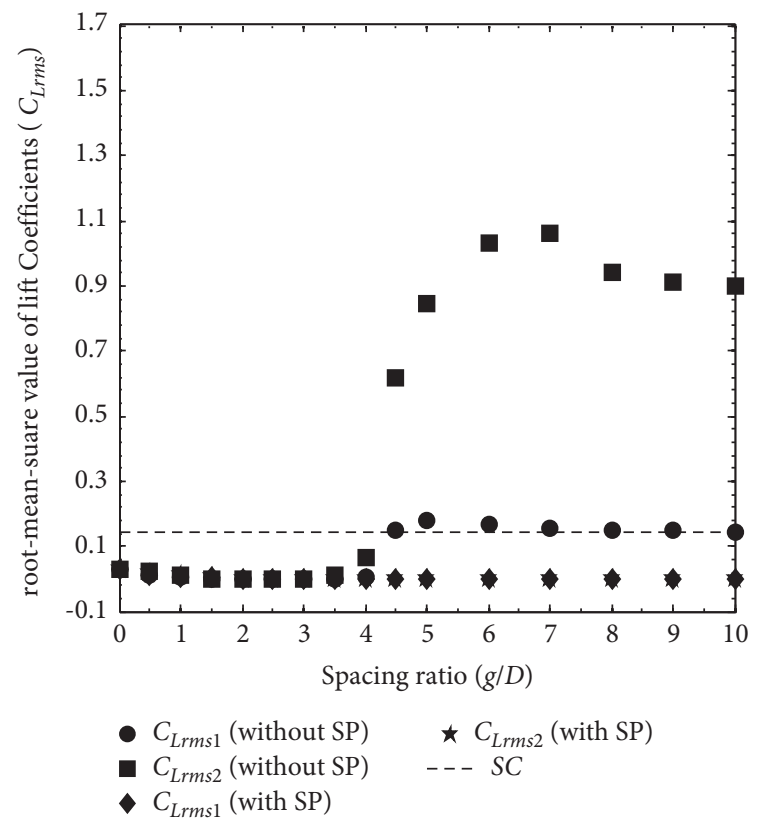

(a)

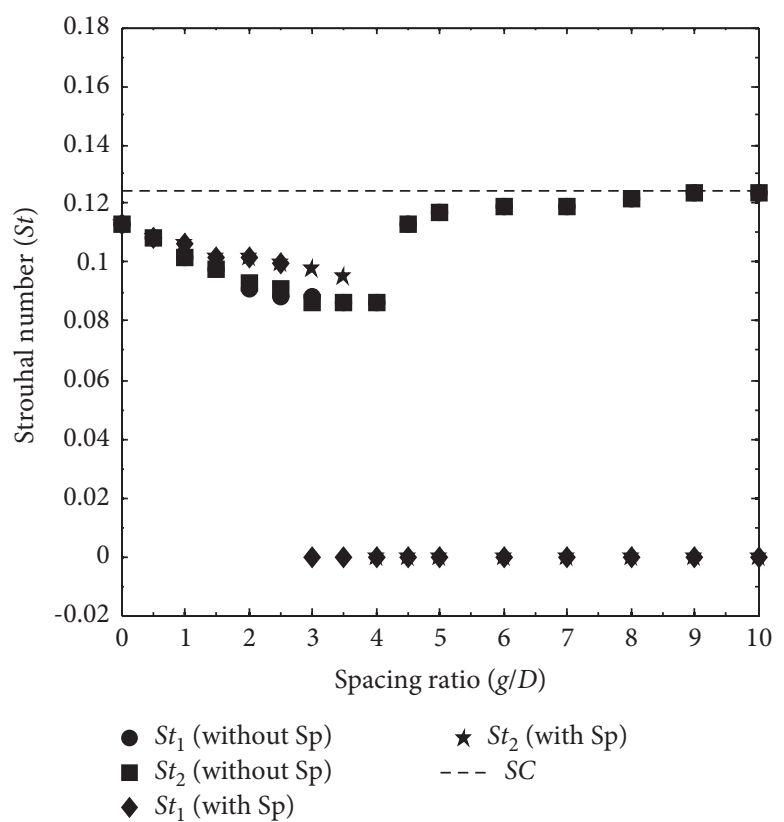

(b)

Figure 13: (a, b). Variation of $C_{L r m s}$ and $S t$ as a function of spacing ratio.

change in $g / D$. The dependency of $C_{L r m s 2}$ is more pronounced at higher spacing ratios. On the increase of length of the splitter plate, the $C_{L r m s 2}$ apparently lower than the value of an isolated SC. The shedding frequency is one of the fluidic parameters which can affect the $C_{2}$ and alter wake dynamics. Similar to the numerical results of Sharman et al.
[30], the St values of the $C_{1}$ and $C_{2}$ are the same with all $g / D$ values considered in this study.

Figure 14 presents the percentage reduction of $C_{D \text { mean }}$ of flow past two tandem cylinders. Regarding the $C_{D \text { mean2 }}$, the control effectiveness increases with increasing the splitter plate length: i.e., $49,53.4,57.2,56.1,62,63.1,67.1,60,53.1$, 


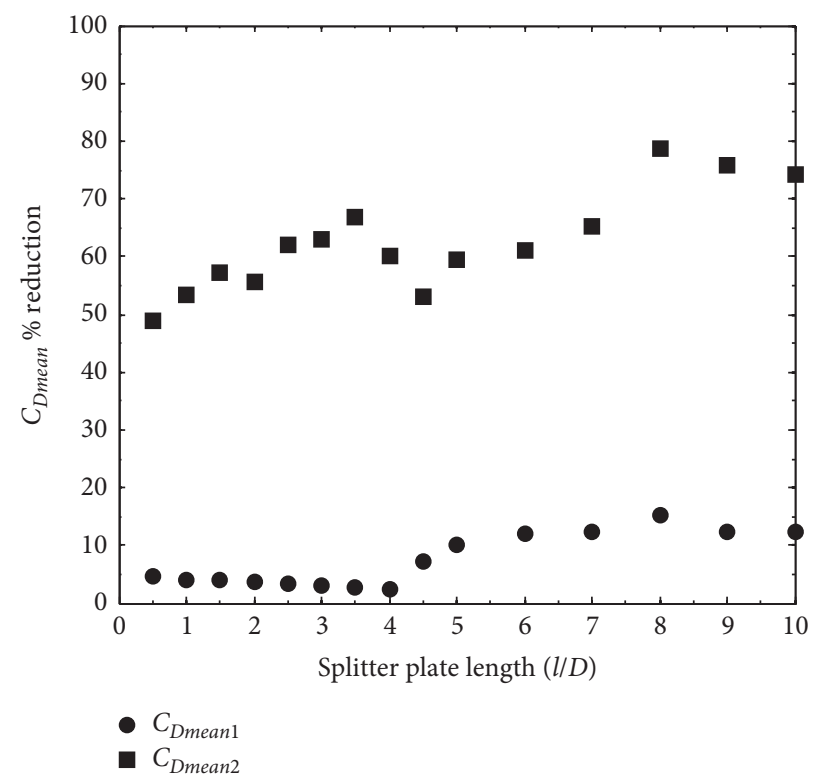

Figure 14: Percentage reduction of $C_{D \text { mean }}$ at different values of $l / D$.

$59.4,61,65.2,79.1,76.1$, and $74.2 \%$ for $l / D=0.5,1,1.5,2,2.5$, $3,3.5,4,4.5,5,6,7,8,9$, and 10 , respectively. The larger splitter plate length will lead to a better control of fluid forces.

\section{Conclusions}

In this article, the numerical simulation for flow past two tandem cylinders in presence of attached splitter plate was carried out at $\mathrm{Re}=100$. This study investigates the drag reduction and the interference among the cylinders at various splitter plate length of $l / D=0.5$ to 10 . The observed flow regimes were divided into five different regimes: single-bluff body, shear layers reattachment, steady, fully developed two-row vortex shedding and fully developed flow regimes. The $C_{D \text { mean2 }}$ was negative when $g /$ $D$ is varied from 0.5 to 4 . We not found fully developed shed vortices between the cylinders in case of single-bluff body and shear layers reattachment flow regimes. The discontinuity is observed in $C_{D \text { rms2 }}$ without splitter plate for the case of $g / D=4.5$ due to transition of flow regime from shear layers reattachment flow regime $(g / D=4)$ to fully developed flow regime $(g / D=4.5)$. The dependency of root-mean-square value of lift coefficient of the downstream cylinder $\left(C_{L \mathrm{rms} 2}\right)$ is more pronounced at higher spacing ratios. Regarding the mean drag coefficient of the downstream square cylinder, the maximum reduction about $79.1 \%$ is observed for $l / D=8$.

\section{Data Availability}

Data will be provided on demand.

\section{Conflicts of Interest}

The authors declare that they have no conflicts of interest.

\section{References}

[1] N. Kondo and D. Matsukuma, "Numerical simulation for aerodynamic characteristics of two circular cylinders in staggered arrangement by a third-order upwind finite element method," International Journal of Computational Fluid Dynamics, vol. 20, no. 8, pp. 1-20, 2006.

[2] H. Sakamoto, H. Hainu, and Y. Obata, "Fluctuating forces acting on two square prisms in a tandem arrangement," Journal of Wind Engineering and Industrial Aerodynamics, vol. 26, no. 1, pp. 85-103, 1987.

[3] A. Azuma, M. Iuchi, I. Watnabe, K. Fujii, K. Sida, and K. Watanabe, "Wind tunnel investigation of buildings and structures," Journal of the Japan Society for Aeronautical and Space Sciences, vol. 22, pp. 209-295, 1974, in Japanese.

[4] W. S. Abbasi, S. U. Islam, and H. Rahman, "Proximity effects on characteristics of flow around three inline square cylinders," Mathematical Problems in Engineering, vol. 2019, 14 pages, 2019.

[5] W. Sarwar Abbasi, S. Ul Islam, L. Faiz, and H. Rahman, "Numerical investigation of transitions in flow states and variation in aerodynamic forces for flow around square cylinders arranged inline," Chinese Journal of Aeronautics, vol. 31, no. 11, pp. 2111-2123, 2018.

[6] S. Mittal, V. Kumar, and A. Raghuvanshi, "Unsteady incompressible flows past two cylinders in tandem and staggered arrangements," International Journal for Numerical Methods in Fluids, vol. 25, no. 11, pp. 1315-1344, 1997.

[7] W. S. Abbasi and S. U. Islam, "Transition from steady to unsteady state flow around two inline cylinders under the effect of Reynolds numbers," Journal of the Brazilian Society of Mechanical Sciences and Engineering, vol. 40, no. 3, p. 168, 2019.

[8] C. K. Vikram, Y. K. Gowda, H. V. Ravindra, and C. G. Gowda, "Numerical simulation of two dimensional unsteady flow past two square cylinders," International Journal of Technology and Engineering System, vol. 2, no. 3, pp. 355-360, 2011.

[9] B. Sharman, F. S. Lien, L. Davidson, and C. Norberg, "Numerical predictions of low Reynolds number flows over two 
tandem circular cylinders," International Journal for $\mathrm{Nu}$ merical Methods in Fluids, vol. 47, no. 5, pp. 423-447, 2005.

[10] C.-H. Liu and J. M. Chen, "Observations of hysteresis in flow around two square cylinders in a tandem arrangement," Journal of Wind Engineering and Industrial Aerodynamics, vol. 90, no. 9, pp. 1019-1050, 2002.

[11] A. Sohankar and A. Etminan, "Forced-convection heat transfer from tandem square cylinders in cross flow at low Reynolds numbers," International Journal for Numerical Methods in Fluids, vol. 60, no. 7, pp. 733-751, 2009.

[12] P. W. Bearman, "Investigation of the flow behind a two-dimensional model with a blunt trailing edge and fitted with splitter plates," Journal of Fluid Mechanics, vol. 21, no. 2, pp. 241-255, 1965.

[13] P. W. Bearman, "The effect of base bleed on the flow behind a two-dimensional model with a blunt trailing edge," Aeronautical Quarterly, vol. 18, no. 3, pp. 207-224, 1967.

[14] M. M. Zdravkovich, "Review and classification of various aerodynamic and hydrodynamic means for suppressing vortex shedding," Journal of Wind Engineering and Industrial Aerodynamics, vol. 7, no. 2, pp. 145-189, 1981.

[15] G. Artana, R. Sosa, E. Moreau, and G. Touchard, "Control of the near-wake flow around a circular cylinder with electrohydrodynamic actuators," Experiments in Fluids, vol. 35, no. 6, pp. 580-588, 2003.

[16] J. H. M. Fransson, P. Konieczny, and P. H. Alfredsson, "Flow around a porous cylinder subject to continuous suction or blowing," Journal of Fluids and Structures, vol. 19, pp. 10311048, 2004.

[17] C. Y. Zhou, L. Wang, and W. Huang, "Numerical study of fluid force reduction on a circular cylinder using tripping rods," Journal of Mechanical Science and Technology, vol. 21, no. 9, pp. 1425-1434, 2007.

[18] Y. J. Chen and C. P. Shao, "Suppression of vortex shedding from a rectangular cylinder at low Reynolds numbers," Journal of Fluids and Structures, vol. 43, pp. 15-27, 2013.

[19] A. Gupta and A. K. Saha, "Suppression of vortex shedding in flow around a square cylinder using control cylinder," $E u$ ropean Journal of Mechanics-B/Fluids, vol. 76, pp. 276-291, 2019.

[20] A. Ahmed, S.-U. Islam, C. Y. Zhou, and R. Manzoor, "Fluid dynamics around three cylinders in presence of small control cylinders," Canadian Journal of Physics, vol. 98, no. 11, pp. 1060-1076, 2020.

[21] K. Kwon and H. Choi, "Control of laminar vortex shedding behind a circular cylinder using splitter plates," Physics of Fluids, vol. 8, pp. 479-486, 1996.

[22] M. Mahbub Alam, H. Sakamoto, and Y. Zhou, "Effect of a $t$ shaped plate on reduction in fluid forces on two tandem cylinders in a cross-flow," Journal of Wind Engineering and Industrial Aerodynamics, vol. 94, no. 7, pp. 525-551, 2006.

[23] E. A. Anderson and A. A. Szewczyk, "Effects of a splitter plate on the near wake of a circular cylinder in 2 and 3-dimensional flow configurations," Experiments in Fluids, vol. 23, no. 2, pp. 161-174, 1997.

[24] D. You, H. Choi, M.-R. Choi, and S.-H. Kang, "Control of flow-induced noise behind a circular cylinder using splitter plates," AIAA Journal, vol. 36, pp. 1961-1967, 1998.

[25] T. Uffinger, S. Becker, and A. Delgado, "Investigations of the flow field around different wall-mounted square cylinder stumps geometries," in Proceedings of the 14th International Symposium on Application of Laser Techniques to Fluid Mechanics, pp. 7-10, Lisbon, Portugal, July 2008.
[26] M. Sukri Mat Ali, C. J. Doolan, and V. Wheatley, "The sound generated by a square cylinder with a splitter plate at low Reynolds number," Journal of Sound and Vibration, vol. 330, no. 15 , pp. $3620-3635,2011$.

[27] Z. Guo, H. Liu, L.-S. Luo, and K. Xu, “A comparative study of the LBE and GKS methods for 2D near incompressible laminar flows," Journal of Computational Physics, vol. 227, no. 10, pp. 4955-4976, 2008.

[28] A. A. Mohammad, Lattice Boltzmann Method: Fundamentals and Engineering Applications with Computer Codes, Springer, Berlin, Germany, Second edition, 2019.

[29] A. Sharma and V. Eswaran, "Heat and fluid flow across a square cylinder in the two-dimensional laminar flow regime," Numerical Heat Transfer, Part A: Applications, vol. 45, no. 3, pp. 247-269, 2004.

[30] R. Mei, D. Yu, W. Shyy, and L.-S. Luo, "Force evaluation in the lattice Boltzmann method involving curved geometry," Physical Review E, vol. 65, pp. 1-28, 2002.

[31] U. Frisch, B. Hasslacher, and Y. Pomeau, "Lattice-gas automata for the Navier-Stokes equation," Physical Review Letters, vol. 56, no. 14, pp. 1505-1508, 1986.

[32] M. Breuer, J. Bernsdorf, T. Zeiser, and F. Durst, "Accurate computations of the laminar flow past a square cylinder based on two different methods: lattice-Boltzmann and finite-volume," International Journal of Heat and Fluid Flow, vol. 21, no. 2, pp. 186-196, 2000.

[33] D. Sumner, S. S. T. Wong, S. J. Price, and M. P. Païdoussis, "Fluid behaviour of side-by-side circular cylinders in steady cross-flow," Journal of Fluids and Structures, vol. 13, no. 3, pp. 309-338, 1999.

[34] S. Dutta, P. K. Panigrahi, and K. Muralidhar, "Effect of orientation on the wake of a square cylinder at low Reynolds numbers," Indian Journal of Engineering and Materials Sciences, vol. 11, no. 6, 2004.

[35] C. Norberg, A. Sohankar, and L. Davidson, "Numerical simulation of unsteady flows around a square two-dimensional cylinder," in Proceedings of the Twelfth Australian Fluid Mechanics Conference, Sydney, Australia, December 1995.

[36] A. Okajima, "Strouhal numbers of rectangular cylinders," Journal of Fluid Mechanics, vol. 123, pp. 379-398, 1982.

[37] T. Igarashi, "Characteristics of the flow around two circular cylinders arranged in tandem: $1^{\text {st }}$ report," Bulletin of JSME, vol. 24, no. 188, pp. 323-331, 1981. 